\title{
Magnetic phases in the one-dimensional Kondo chain on a metallic surface
}

\author{
Alejandro M. Lobos, ${ }^{1}$ Miguel A. Cazalilla, ${ }^{2,3,4}$ and Piotr Chudzinski ${ }^{5}$ \\ ${ }^{1}$ Condensed Matter Theory Center, Department of Physics, University of Maryland, College Park, Maryland 20742-4111, USA \\ ${ }^{2}$ Donostia International Physics Center (DIPC), Manuel de Lardizábal 4, E-20018 San Sebastián, Spain \\ ${ }^{3}$ Graphene Research Centre, National University of Singapore, 6 Science Drive 2, Singapore 117546 \\ ${ }^{4}$ Centro de Física de Materiales (CFM), Centro Mixto CSIC-UPV/EHU, Edificio Korta, Avenida de Tolosa 72, E-20018 San Sebastián, Spain \\ ${ }^{5}$ DPMC-MaNEP, University of Geneva, 24 Quai Ernest-Ansermet CH-1211 Geneva, Switzerland
}

(Received 9 March 2012; published 30 July 2012; corrected 1 August 2012)

\begin{abstract}
We study the low-temperature properties of a one-dimensional spin-1/2 chain of magnetic impurities coupled to a (normal) metal environment by means of anisotropic Kondo exchange. In the case of easy-plane anisotropy, we obtain the phase diagram of this system at $T=0$. We show that the in-plane Kondo coupling destabilizes the Tomonaga-Luttinger phase of the spin chain, and leads to two different phases: (i) At strong Kondo coupling, the spins in the chain form Kondo singlets and become screened by the metallic environment, and (ii) at weak and intermediate Kondo coupling, we find a novel dissipative phase characterized by diffusive gapless spin excitations. The two phases are separated by a quantum critical point of the Wilson-Fisher universality class with dynamical exponent $z \simeq 2$.
\end{abstract}

DOI: 10.1103/PhysRevB.86.035455

PACS number(s): 75.10.Pq, 75.20.Hr, 75.40.-s

\section{INTRODUCTION}

Magnetic structures of atomic size provide the smallest solid-state systems in which it is possible to store (quantum) information. ${ }^{1}$ The possibility of building and manipulating such atomic-scale magnetic structures has been demonstrated in recent experiments using scanning tunneling microscopy (STM), ${ }^{1-5}$ a fact that paves the way for the realization of spin devices of nanoscopic size.

Besides the interest in applications, magnetic systems at the nanoscale constitute an excellent playground to address fundamental questions in condensed matter physics. For instance, magnetic impurities inside a metallic host have shown clear evidences of the Kondo effect in the scanning tunneling spectra (STS). ${ }^{6-8}$ The Kondo effect (i.e., the spin compensation of a localized magnetic moment by conduction electrons in a metal) is one of the most paradigmatic phenomena in many-body physics. ${ }^{9}$ On the other hand, magnetic atoms inside a metal can interact nonlocally via the electronic medium through the Ruderman-Kittel-Kasuya-Yosida (RKKY) exchange coupling, ${ }^{10}$ which is responsible for the magnetic properties of many heavy-fermion materials ${ }^{11}$ and for the giant-magnetoresistance effect in layered magnetic heterostructures. ${ }^{12}$ Direct evidence of RKKY interaction at the atomic scale (i.e., among pairs of magnetic Fe or Co atoms) has been reported recently in STM experiments. ${ }^{4,13}$ Due to the ability to control the distance between magnetic atoms using the STM tip, the RKKY interaction can be, in principle, tuned from ferromagnetic to antiferromagnetic, and this oscillating feature has been clearly revealed in recent experiments showing spin-polarized STM maps. ${ }^{4}$

By depositing atoms one by one, STM also enables one to build magnetic structures where the dimensionality is gradually changed from the zero-dimensional (0D) limit to the one-dimensional (1D) case. In particular, linear arrays of up to 10 magnetic $\mathrm{Mn}$ atoms ${ }^{3}$ and, more recently, antiferromagnetic chains made of eight and more $\mathrm{Fe}$ atoms ${ }^{14}$ have been assembled and analyzed with STM and inelastic electron tunnel spectroscopy (IETS). From the theoretical point of view, these low-dimensional magnetic systems are of interest due to the prominent effect of quantum fluctuations, which inhibit magnetic order and, at low temperatures, lead to quantum phases with exotic properties. ${ }^{15}$

Motivated by the experimental progress described above, in this work we study a $1 \mathrm{D}$ chain of spin $S=1 / 2$ magnetic atoms (i.e., Kondo impurities) embedded in (or, rather, deposited on) a host such like a metallic surface (cf. Fig. 1). The magnetic atoms are coupled to each other and to the metallic host by means of an anisotropic exchange. The anisotropic coupling between the magnetic atoms may be regarded as a consequence of, for example, a Dzyaloshinskii-Moriya interaction resulting from the spin-orbit coupling in the host. From a different perspective, this system also provides a realization of a 1D dissipative system, where the interplay between quantum fluctuations and dissipation can have important consequences for the quantum phase diagram. ${ }^{16}$ Examples of other physical realizations of $1 \mathrm{D}$ dissipative systems can be found in, for example, resistively shunted 1D Josephson junction arrays, ${ }^{17-22}$ Tomonaga-Luttinger liquids coupled to dissipative baths, ${ }^{23-25}$ superconducting wires coupled to diffusive metals, ${ }^{24,26,27}$ and 1D ultracold atom gases embedded in a Bose-Einstein condensate. ${ }^{28}$

Using a host of analytical methods, which include bosonization and renormalization group methods, we investigate the effects of the metallic environment on the spin chain. At $T=0$, we predict that the Tomonaga-Luttinger liquid (TLL) phase of the impurity spin chain will be destabilized by the (in-plane) Kondo interaction, $J_{K}^{\perp}$, with the metallic host. Turning on $J_{K}^{\perp}$, yields a dissipative phase, whose ground state exhibits long-range order (LRO) of the in-plane magnetization. Upon increase of $J_{K}^{\perp}$, we predict a quantum phase transition towards a disordered Kondo-singlet phase, where the spins of the 1D chain are locally screened by the host electrons, and the LRO along the chain is destroyed.

This work is organized as follows. In Sec. II we present the model for the anisotropic spin chain coupled to a normal metal, in Sec. III we show our main results, divided into 


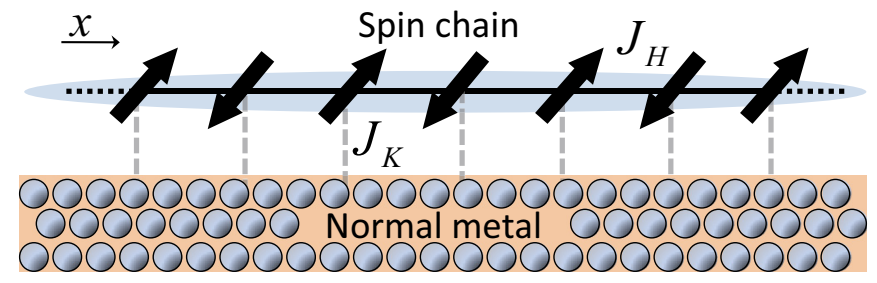

FIG. 1. (Color online) Scheme of the system representing a $S=1 / 2$ spin chain coupled to a metallic environment through the Kondo Hamiltonian. The presence of the metallic surface breaks the rotational and inversion symmetry, inducing in-plane anisotropy.

weak-coupling (Sec. III A) and strong-coupling (Sec. III B) treatment of the Kondo Hamiltonian, in Sec. IV we present a summary and conclusions, and finally, the details of the calculations are in Appendixes A and B.

\section{MODEL}

The Hamiltonian of the spin-chain system embedded in a metallic host can be split into three terms:

$$
\mathcal{H}=\mathcal{H}_{C}+\mathcal{H}_{F}+\mathcal{H}_{K},
$$

where $\mathcal{H}_{C}=\sum_{i j, \alpha, \beta} \tilde{J}_{H}^{\alpha \beta}(i j) \tilde{S}_{i}^{\alpha} \tilde{S}_{j}^{\beta}$ describes the (static) interactions between the magnetic atoms in the chain, $\mathcal{H}_{K}$ accounts for the coupling of the chain to the metallic host, and $\mathcal{H}_{F}$ describes the host electrons. In a real experimental system the physical origin of the term $\mathcal{H}_{C}$ is not always easy to identify. Although in general the RKKY interaction is believed to mediate magnetic interactions between magnetic adatoms deposited on metals [e.g., Co adatoms deposited on the top of $\mathrm{Cu}(100)$ surfaces ${ }^{4,13,29}$, more complicated situations, requiring detailed first-principles calculations to identify the source of magnetic coupling, might arise. Determining the physical origin of $\mathcal{H}_{C}$ is, however, beyond the scope of the present work, and will not be relevant for our purposes in what follows. Here we assume quite generally that the coupling $\tilde{J}_{H}^{\alpha \beta}(i j)$ decays with the distance between impurities, allowing one to truncate the interaction at nearest neighbors. Thus, we consider the following model:

$$
\begin{aligned}
\mathcal{H}_{C}= & \sum_{j} \frac{\tilde{J}_{H}^{\perp}}{2}\left(\tilde{S}_{j+1}^{+} \tilde{S}_{j}^{-}+\tilde{S}_{j}^{-} \tilde{S}_{j+1}^{+}\right)+\tilde{J}_{H}^{z} \tilde{S}_{j}^{z} \tilde{S}_{j+1}^{z} \\
& +\sum_{j} D \hat{\mathbf{z}} \cdot\left(\tilde{\mathbf{S}}_{j} \times \tilde{\mathbf{S}}_{j+1}\right) .
\end{aligned}
$$

For definiteness, we assume here that the index $j$ runs along the $\hat{x}$ axis (cf. Fig. 1). The last term in Eq. (2) is a DzyaloshinskiiMoriya (DM) interaction, which results from the spin-orbit coupling of the electrons at the surface of the metallic host. The symmetry conditions for the DM interaction to exist are rarely met in the bulk of typical metals. However, for an impurity chain that lies on a metallic surface where inversion symmetry is broken, a DM interaction term is in principle expected. ${ }^{30}$ The above spin-chain model, Eq. (2), can be mapped onto a 1D Heisenberg-Ising (XXZ) $S=1 / 2$ chain by the following transformation: ${ }^{31}$

$$
\begin{gathered}
S_{j}^{ \pm}=e^{i \eta j} \tilde{S}_{j}^{ \pm}, \\
S_{j}^{z}=\tilde{S}_{j}^{z},
\end{gathered}
$$

where $\eta=\tan ^{-1}\left(D / \tilde{J}_{H}\right)(0 \leqslant \eta \leqslant \pi / 2)$. Thus, Eq. (2) becomes

$$
\mathcal{H}_{X X Z}=\sum_{j} \frac{J_{H}^{\perp}}{2}\left(S_{j}^{+} S_{j+1}^{-}+S_{j}^{-} S_{j+1}^{+}\right)+J_{H}^{z} S_{j}^{z} S_{j+1}^{z},
$$

with $J_{H}^{\perp}=\tilde{J}_{H}^{\perp} / \cos \eta$ and $J_{H}^{z}=\tilde{J}_{H}^{z}$. Although the DM interaction in metallic surfaces is $\operatorname{small}^{30}$ (i.e., $D / J_{H} \ll 1$ ), it can dramatically affect the properties of the spin chain. In particular, if the initial impurity-chain Hamiltonian (2) is isotropic (i.e., $\tilde{J}_{H}^{\perp}=\tilde{J}^{z}$ ), the above transformation (3) maps it onto an $X X Z$ spin chain with easy-plane anisotropy: $\left|J_{H}^{\perp} / J_{H}^{z}\right|=(\cos \eta)^{-1}>1$. Therefore, from here on, we shall focus on the case of the $X X Z$ spin chain with in-plane anisotropy. Under these assumptions, it is well known that the low-energy sector of Hamiltonian Eq. (5) maps onto the $X Y$ model, whose spectrum is described in terms of gapless spinon modes and exhibits power-law magnetic correlations ${ }^{15,32}$ $\left\langle S_{j}^{+} S_{j+n}^{-}\right\rangle \sim|n|^{-v}$.

The coupling between the $X X Z$ chain and the metal is described by the following anisotropic Kondo exchange interaction: ${ }^{9}$

$$
\begin{aligned}
\mathcal{H}_{K}= & \sum_{j} \frac{1}{2} J_{K}^{z} S_{j}^{z}\left[c_{\uparrow}^{\dagger}\left(\mathbf{R}_{j}\right) c_{\uparrow}\left(\mathbf{R}_{j}\right)-c_{\downarrow}^{\dagger}\left(\mathbf{R}_{j}\right) c_{\downarrow}\left(\mathbf{R}_{j}\right)\right] \\
& +\frac{J_{K}^{\perp}}{2} e^{i \eta j} S_{j}^{+} c_{\downarrow}^{\dagger}\left(\mathbf{R}_{j}\right) c_{\uparrow}\left(\mathbf{R}_{j}\right)+\text { H.c. },
\end{aligned}
$$

where Eqs. (3) and (4) have been used. In Eq. (6) every spin $\mathbf{S}_{j}$ in the chain is coupled to the fermionic spin density of the bath. The operator $c_{\sigma}^{\dagger}(\mathbf{R})$ creates an electron with spin projection $\sigma$ at position $\mathbf{R}=(x, y, z)$ in the metal, and for a chain site $\mathbf{R}_{j}=\left(j a_{0}, 0,0\right)$ with $a_{0}$ the lattice parameter of the impurity chain. Here we also assume an anisotropic Kondo interaction with in-plane anisotropy $\left|J_{K}^{\perp} / J_{K}^{z}\right|>1$.

Finally, the dynamics of the electrons in the metallic host is described in terms of Landau quasiparticles,

$$
\mathcal{H}_{F}=\sum_{\mathbf{k}, \sigma} \epsilon_{\mathbf{k}} c_{\sigma}^{\dagger}(\mathbf{k}) c_{\sigma}(\mathbf{k})+\cdots,
$$

where $\epsilon_{\mathbf{k}}$ is the electron dispersion and $c_{\sigma}(\mathbf{k}) \equiv$ $\int d^{3} \mathbf{R} e^{i \mathbf{k} \cdot \mathbf{R}} c_{\sigma}(\mathbf{R})$ is the Fourier transform of the annihilation operator $c_{\sigma}(\mathbf{R})$. The dots in Eq. (7) stand for additional terms, such as spin-obit interactions, whose form needs not be specified. An important parameter describing the properties of the metallic host is the Fermi wave vector $k_{F}$. In a real experimental situation, although the magnetic nanostructure is built on the top of the 2D metallic surface, $k_{F}$ might have a 3D character due to a nonvanishing overlap with the bulk conduction states in the metal. ${ }^{8}$

Intuitively, the Hamiltonian Eq. (1) encodes a competition between the Heisenberg interaction, which favors correlations along the spin chain, and the Kondo coupling, which tends to screen locally the impurity-spins and promotes a nonmagnetic ground state. Our description is therefore very similar to the well-known case of heavy-fermion materials described by the (3D) Kondo lattice model, where this competition between the Kondo and RKKY interactions is believed to be responsible for the unusual quantum critical properties and complex magnetic phase diagram..$^{9,33,34}$ In that context, it has 
been shown (see, e.g., Ref. 33) that although both interactions are due to coupling of spins with the same Fermi sea, they can be treated separately since the RKKY interaction originates from electronic states deep inside the Fermi sea, while the Kondo effect is purely a Fermi-surface effect. In our particular case the interplay between Heisenberg and Kondo interaction is quite subtle due to the additional effects of plane anisotropy and reduced dimensionality of the spin chain, leading to a counterintuitive cooperation effect in a certain regime of parameters and to a nontrivial phase diagram at $T=0$ (cf. Fig. 3 below).

One should note that our model significantly differs from previous approaches to the 1D Heisenberg-Kondo model $(1 \mathrm{DHKM})^{35-37}$ or the 1D Kondo-lattice model (1DKLM). ${ }^{38-42}$ Those works assumed entirely 1D situations in which a 1D electron gas acting as a host is coupled either with a 1D spin chain (1DHKM) or with a set of independent (0D) spins (1DKLM), a $J_{H}=0$ limit of the 1DHKM. In those works the $1 \mathrm{D}$ character of the bath allowed not only for a straightforward application of 1D methods (e.g., bosonization or DMRG), but it also contained hidden constrains like spin-charge separation or a reduction of the Fermi surface only to two points (thus allowing for a very limited number of scattering channels). In particular, it was shown that the magnetic scattering on spin impurities opens a spin gap in the spectrum of the 1D fermionic bath. ${ }^{36,38,39}$ This result is in clear contrast with our assumption in Eq. (7), where we take the higher-dimensional bath to be unaffected by low dimensional impurities. The principal aim of those previous works was also different (i.e., to establish analogies with phases present in heavy-fermion compounds), as clearly expressed in a review article. ${ }^{40}$

In contrast, our model is closer to the experimental situations described in Sec. I, where the spin chain is embedded in or lies on a higher-dimensional metallic host with a large Fermi surface and large Fermi energy. Consequently, in our work many of the above-mentioned constraints existing in the entirely $1 \mathrm{D}$ geometry are released. The reason why this drastically changes the physics of the problem results from the absence of the Nozières' "exhaustion" problem: ${ }^{43}$ In our case, a higher-dimensional bath ensures the presence of enough conduction electrons to screen the magnetic impurities on the scale $\sim k_{F}^{-1}$ (cf. Sec. III B below for details). One should keep in mind that taking the higher-dimensional bath is at the core of this paper and is in fact crucial to justify the local approximation in Sec. III B (i.e., independent local-bath approximation).

In the following, we explore the quantum critical properties of model Eq. (1) at low energies and for different regimes of parameters $J_{H}^{\perp}, J_{H}^{z}, J_{K}^{\perp}$, and $J_{K}^{z}$. Throughout we shall use units where $\hbar=1$.

\section{RESULTS}

\section{A. Weak coupling scaling analysis}

In the regime where the Heisenberg coupling $J_{H}^{\perp}$ dominates (i.e., $J_{H}^{\perp}$ is much larger than $J_{K}^{\perp}$ and $J_{K}^{z}$ ), a good starting point is to treat the Kondo coupling $\mathcal{H}_{K}$ [cf. Eq. (6)] as a small perturbation to the isolated spin-chain Hamiltonian $\mathcal{H}_{X X Z}$ [cf. Eq. (5)]. In this case, Hamiltonian Eq. (5) can be studied within the framework of Abelian bosonization, ${ }^{15}$ which allows one to map it onto the continuous $X Y$ Hamiltonian,

$$
\mathcal{H}_{X X Z}=\frac{1}{2 \pi} \int d x\left[\frac{u}{K}(\nabla \Phi)^{2}+u K(\nabla \Theta)^{2}\right]+\cdots
$$

Here $\Theta(x), \Phi(x)$ are conjugate canonical fields obeying the usual commutation relations $\left[\Theta(x), \nabla \Phi\left(x^{\prime}\right)\right]=i \pi \delta\left(x-x^{\prime}\right)$. These fields are continuous in the scale of $a_{0}$, the original lattice spacing in the chain, and are related to the original spin operators by (cf. Ref. 15):

$$
\begin{array}{r}
S_{j}^{ \pm}=a_{0} S^{ \pm}\left(x_{j}\right)=\frac{e^{\mp i \Theta\left(x_{j}\right)}}{\sqrt{2 \pi}}\left[e^{i x_{j} \pi / a_{0}}+\cos 2 \Phi\left(x_{j}\right)\right], \\
S_{j}^{z}=a_{0} S^{z}\left(x_{j}\right)=-\frac{a_{0}}{\pi} \nabla \Phi\left(x_{j}\right)+\frac{e^{i x_{j} \pi / a_{0}}}{\sqrt{\pi}} \cos 2 \Phi\left(x_{j}\right),
\end{array}
$$

where $x_{j}=j a_{0}$ is the position of the $j$ th spin. The model (8) describes 1D gapless spinon excitations in the transverse direction propagating with velocity $u$, and is parametrized by the dimensionless Luttinger parameter, ${ }^{44,45}$ $K=\left[\frac{2}{\pi} \arccos \left(-J_{H}^{z} / J_{H}^{\perp}\right)\right]^{-1}$, which determines the decay of the correlation functions in the chain, for example, $\left\langle S^{+}(x) S^{-}(0)\right\rangle \sim|x|^{-1 / 2 K}$. The isotropic Heisenberg model is recovered for the particular value $K=1 / 2$. As discussed above, in this work we focus on the regime of easy-plane anisotropy, which corresponds to the condition that $K>1 / 2$. The ellipsis in (8) stands for additional perturbations, such as the sine-Gordon term $\sim \cos 4 \Phi(x)$, which are irrelevant in the renormalization-group (RG) sense for $K>\frac{1}{2}$ and will be neglected.

The continuum limit of the Kondo Hamiltonian, Eq. (6), reads

$$
\begin{aligned}
\mathcal{H}_{K}= & \int d x \frac{J_{K}^{z}}{k_{F}^{3}} S^{z}(x) s^{z}(x) \\
& +\int d x \frac{J_{K}^{\perp}}{2 k_{F}^{3}}\left[e^{i q_{D M} x} S^{+}(x) s^{-}(x)+\text { H.c. }\right],
\end{aligned}
$$

where we have defined wave vector $q_{\mathrm{DM}} \equiv \eta / a_{0}$ associated with the DM interaction, and introduced the factors of the Fermi momentum $k_{F}$ in order for the Kondo couplings to have dimensions of energy. We have also defined the spin-density operator of the host electrons as

$$
s^{a}(\mathbf{R}) \equiv \sum_{\sigma, \sigma^{\prime}} c_{\sigma}^{\dagger}(\mathbf{R})\left[\frac{\boldsymbol{\sigma}^{a}}{2}\right]_{\sigma, \sigma^{\prime}} c_{\sigma^{\prime}}(\mathbf{R}),
$$

with $\sigma^{a}(a=x, y, z)$ the Pauli matrices. From Eqs. (10) and (11) we note that the operator $\nabla \Phi(x)$ couples to the uniform component of the spin density in the electron gas $s_{u}^{z}(x) \equiv s^{z}(x)$, and the operator $\cos 2 \Phi(x)$ couples to the staggered component $s_{s}^{z}(x)=e^{i x \pi / a_{0}} s^{z}(x)$. On the other hand, the operator $e^{-i \Theta\left(x_{j}\right)}$ couples to the staggered magnetization $s_{s}^{-}(x)=e^{i x \pi / a_{0}} s^{-}(x)$. These contributions to Eq. (11) have different scaling dimensions, and we treat them independently in the following analysis.

Next, we assess the stability of the TLL phase, which is described by Hamiltonian in Eq. (8). To this end, we consider the leading order corrections to the free energy per unit of length in the impurity spin chain. The technical details of this calculation can be found in Appendix A. We shall 
not pursue the stability analysis beyond the leading order in this work, as our focus here is on the phase diagram in the $K>1 / 2$ (i.e., $J_{H}^{\perp}>J_{H}^{z}$ ) regime, for which, as the following discussion demonstrates, there is only one relevant Kondo coupling, namely $J_{K}^{\perp}$. A more complete analysis will be reported elsewhere. ${ }^{46}$ To leading order in the Kondo couplings, for temperatures $T \ll J_{H}^{\perp}$, we find

$$
\begin{aligned}
\frac{\Delta F}{L}= & g_{z, u}^{2} \frac{K A_{u}^{z}}{2^{6} \pi^{2} u^{2} k_{F}} T^{3} \\
& +g_{z, s}^{2} \frac{A_{s}^{z}}{2^{4} k_{F} a_{0}^{2} \pi^{3}}\left(\frac{\pi a_{0}}{u}\right)^{2 K} T^{1+2 K} \\
& +g_{\perp, s}^{2} \frac{A_{s}^{\perp}}{2^{5} k_{F} a_{0}^{2} \pi^{3}}\left(\frac{\pi a_{0}}{u}\right)^{1 / 2 K} T^{1+1 / 2 K},
\end{aligned}
$$

where $L \rightarrow \infty$ is the impurity chain length and the dimensionless couplings $g_{z, u} \equiv J_{K}^{z} / v_{F} k_{F}, g_{z, s} \equiv J_{K}^{z} / v_{F} k_{F}$, and $g_{\perp, s} \equiv$ $J_{K}^{\perp} / v_{F} k_{F}$ have been introduced; $A_{u}^{z}, A_{s}^{z}$, and $A_{s}^{\perp}$ are nonuniversal numerical coefficients. As explained in Appendix A, Eqs. (13)-(15) reflect the fact that the metallic host exhibits an Ohmic spectrum of magnetic excitations over a broad range of momentum transfer along the spin-chain direction. This means that the metallic host contributes to $\Delta F$ effectively as if it was a local fermionic bath, that is, the spin-spin correlation function, $\chi^{a b}\left(\mathbf{R}_{1}-\mathbf{R}_{2}, \tau_{1}-\tau_{2}\right) \equiv-\left\langle T_{\tau} s^{a}\left(\mathbf{R}_{1}, \tau_{1}\right) s^{b}\left(\mathbf{R}_{2}, \tau_{2}\right)\right\rangle_{0}$, behaves effectively as a "local" function $\chi^{a b}\left(\mathbf{R}_{1}-\mathbf{R}_{2}, \tau_{1}-\tau_{2}\right) \propto$ $\delta_{\mathbf{R}_{1}, \mathbf{R}_{2}} /\left(\tau_{1}-\tau_{2}\right)^{2}$ (cf. Sec. A). We will come back to this point in Sec. III B.

The stability of the TLL phase with respect to the perturbation $\mathcal{H}_{K}$ can be now assessed by comparing the scaling with temperature of $\Delta F$ and the free energy of the spin-chain chain $F_{0}$, when decoupled from the environment, which behaves as ${ }^{15,47} F_{0} \sim T^{2}$. Thus we look for divergences in the perturbative corrections to $\Delta F / F_{0}$ as the temperature is gradually decreased towards the ground state (i.e., $T=0$ ). From (15), it can be seen that the term $\propto g_{\perp, s}^{2}$ yields a divergent contribution to $\Delta F / F_{0}$, which signals an instability of the TLL phase.

To make contact with the renormalization group (RG), we shall define the effective couplings $g_{z, u}(\ell) \equiv g_{z, u} e^{-(1 / 2) \ell}$, $g_{z, s}(\ell) \equiv g_{z, s} e^{(1 / 2-K) \ell}$, and $g_{\perp, s}(\ell) \equiv g_{\perp, s} e^{(1 / 2-1 / 4 K) \ell}$, where $\ell \equiv \ln \left(\Lambda_{0} / T\right)$ and $\Lambda_{0} \sim J_{H}^{\perp}$ is the high-energy cutoff for the effective low-energy description of the spin chain in terms of Eq. (8). Decreasing the temperature a bit towards the ground state (i.e., $T=0$ ) can be regarded as an infinitesimal change of $\ell \rightarrow \ell+\delta \ell$, and the corresponding change ("flow") of the effective couplings can be described by the following set of differential equations:

$$
\begin{gathered}
\frac{d g_{z, u}(\ell)}{d \ell}=-\frac{1}{2} g_{z, u}(\ell), \\
\frac{d g_{z, s}(\ell)}{d \ell}=\frac{1}{2}(1-2 K) g_{z, s}(\ell), \\
\frac{d g_{\perp, s}(\ell)}{d \ell}=\frac{1}{2}\left(1-\frac{1}{2 K}\right) g_{\perp, s}(\ell) .
\end{gathered}
$$

Alternatively, we can regard these equations as describing the change in effective (dimensionless) couplings of an equivalent (coarse-grained) system with a reduced high-energy cutoff $\Lambda(\ell)=e^{-\ell} \Lambda_{0}$. This interpretation means that for in-plane anisotropy where $K>1 / 2$, the couplings $g_{z, u}(\ell)$ and $g_{z, s}(\ell)$ decrease as the system is coarse grained by integrating out the high-energy degrees of freedom and become irrelevant (in the RG sense). In other words, the terms in $\mathcal{H}_{K}$ proportional to those couplings yield subleading corrections and therefore can be neglected as $T \rightarrow 0$. On the other hand, $g_{\perp, s}(\ell)$ is a relevant (in the RG sense) perturbation, which, as $T \rightarrow 0$ yields a dominant correction and destabilizes the TLL phase of the spin chain described by Eq. (8). Note that both $g_{z, s}(\ell)$ and $g_{\perp, s}(\ell)$ have the same scaling dimension at the Heisenberg point $(K=1 / 2)$, where they are marginally relevant, and a higher order perturbative analysis is required to fully assess the stability of the TLL phase. ${ }^{46}$

Note that the physics described by Eqs. (16)-(18) can be mimicked by an infinite set of fermionic baths, each bath being locally coupled to only one impurity spin (cf. Fig. 2), which yields a local (i.e., momentum independent) Ohmic spin response: for example, $\chi^{+-}(x, \tau), \chi^{z z}(x, \tau) \sim \delta(x) / \tau^{2}$. As will be discussed in the next section, this model allows us to treat the relevant Kondo coupling $J_{K}^{\perp}$ in a nonperturbative way. In particular, it captures the important (nonperturbative) feature that the magnetic moment of the impurities will be fully screened by the metallic environment at large $J_{K}^{\perp} \gg J_{H}^{\perp}$. In the above analysis, the need for a nonperturbative treatment is evidenced by the fact that even an infinitesimal value of $J_{K}^{\perp}$ will destabilize the TLL phase for in-plane anisotropy $(K>1 / 2)$. However, different from the single-impurity Kondo problem, we will see below that the RG flow does not proceed from the TLL phase into a strong coupling Kondo-screened phase in a straightforward manner, but rather, another phase of dissipative nature intervenes between the TLL and the Kondo phase.

\section{B. Strong coupling analysis}

\section{Derivation of an effective $1 D$ model}

As mentioned before, when $J_{K}^{\perp}$ flows to strong coupling, the perturbative RG approach used in Sec. III A is no longer valid, and we need to study the physical properties of the spin chain in a different way. The approach used in this section is motivated by the following arguments: (i) The analysis made in the previous section and in the Appendix A indicates that the most relevant coupling of the spin chain to the metal arises from the local sector of the spin response in the metal and, (ii) at strong coupling, for a 2D or 3D host, the interference of two Kondo screening clouds belonging

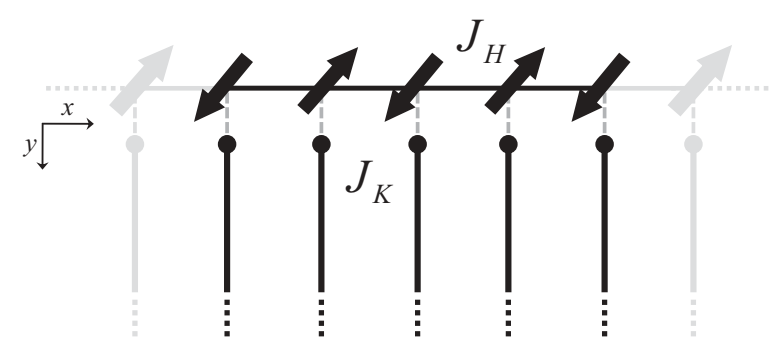

FIG. 2. Schematic diagram of the spin chain coupled to the metallic host, after the independent-bath approximation. Due to the locality of the dynamic spin susceptibility, which decays on distances of the order of $\sim k_{F}^{-1}$, the interference of Kondo-screening clouds is negligible in the limit $k_{F} a_{0} \gg 1$. 
to spins located at a distance $a_{0}$ decays rapidly when $a_{0}$ is of the order of a few Fermi wavelengths (i.e., $\sim k_{F}^{-1}$ ). ${ }^{48-50}$ In our case, the higher dimensionality of the metallic host as compared to the spin chain allows one to rule out the well-known exhaustion problem ${ }^{51}$ and the spins in the chain can be considered as independently screened in the regime $k_{F} a_{0} \gtrsim 1$. Experimentally, this is confirmed by the behavior of the STS Fano line shapes in magnetic Co atoms deposited on $\mathrm{Cu}(100)$ and separated by distances $a_{0}>8 \AA$, which are identical to the single-impurity STS line shapes. ${ }^{13}$ This is an important difference with respect to the strictly 1D Kondo-lattice model, where the single Kondo-impurity limit is reached only at distances $a_{0} \gg \xi_{K} \sim v_{F} / T_{K} .{ }^{38}$ Since the Kondo temperature is an exponentially small energy scale, in the purely $1 \mathrm{D}$ geometry the single-impurity regime is only reached at extremely dilute impurity-spin concentrations. ${ }^{49,50}$

In our model, the larger dimensionality of the metallic host suggests that, in the regime where $k_{F} a_{0} \gtrsim 1$, it is reasonable to approximate the Hamiltonian $\mathcal{H}_{F}$ in Eq. (7) by a set of independent fermionic baths (i.e., semi-infinite 1D chains; cf. Fig. 2) coupled to each spin in the chain $\mathbf{S}_{i}$, that is,

$$
\mathcal{H}_{F} \simeq-t \sum_{i j \sigma} c_{i, j, \sigma}^{\dagger} c_{i, j+1, \sigma}+\text { H.c. }
$$

where the index $i(j)$ runs along the $\hat{x}(\hat{y})$ axis. This is a minimal model that captures the competition between Kondo and Heisenberg interactions. Note that this approximation is consistent with the local limit of Sec. III A.

In the regime $k_{F} a_{0} \ll 1$, an electron at the Fermi energy cannot distinguish between neighboring individual spins. ${ }^{48,52}$ Therefore, in that case assuming a local fermionic bath for each impurity may appear to be a rather uncontrolled approximation. However, whether this approximation breaks down or not in that regime will be the subject of future research. ${ }^{46}$ Actually, we may regard the model resulting form Eq. (19) as a good starting point for such further investigations.

The advantage of the independent-bath approximation Eq. (19) is that it allows one to use powerful analytical methods which have been applied successfully to describe the single Kondo-impurity problem. In the following, we implement the Abelian bosonization approach to the Kondo problem. ${ }^{15,47,53-55}$ To avoid confusion with the previous Sec. III A, note that here bosonization is implemented to describe the fermionic $1 D$ chains, and not the spin chain. At low energies the Hamiltonians $\mathcal{H}_{F}$ and $\mathcal{H}_{K}$ become in the bosonic representation, ${ }^{55}$

$$
\begin{gathered}
\mathcal{H}_{F}=\sum_{i, \nu=\{c, s\}} \frac{v_{F}}{4 \pi} \int_{-\infty}^{\infty} d y\left(\nabla \phi_{i, v}^{R}(y)\right)^{2}, \\
\mathcal{H}_{K}=\sum_{i}-\frac{2 \delta_{s}}{\pi \rho_{0}} S_{i}^{z} \frac{\nabla \phi_{i, s}^{R}(0)}{\sqrt{2} \pi} \\
+\frac{J_{K}^{\perp} b_{0}}{2}\left[S_{i}^{+} \frac{e^{-i \sqrt{2} \phi_{i, s}^{R}(0)}}{2 \pi b_{0}}+S_{i}^{-} \frac{e^{i \sqrt{2} \phi_{i, s}^{R}(0)}}{2 \pi b_{0}}\right],
\end{gathered}
$$

where the chiral fields $\phi_{i, c}^{R}(y), \phi_{i, s}^{R}(y)$ obey the commutation relations $\left[\phi_{i, v}^{R}(y), \phi_{j, \eta}^{R}\left(y^{\prime}\right)\right]=i \pi \operatorname{sign}\left(y-y^{\prime}\right) \delta_{i, j} \delta_{v, \eta}$, and are related to charge and spin density fluctuations through the relations $\rho_{i}(y)=-\frac{1}{\pi} \nabla \phi_{i, c}^{R}(y)$ and $s_{i}(y)=-\frac{1}{\pi} \nabla \phi_{i, s}^{R}(y)$, respectively. ${ }^{15}$ In Eq. (20) $v_{F}$ is the Fermi velocity, and in Eq. (21) $\delta_{s}=\tan ^{-1}\left(\pi \rho_{0} J_{K}^{z} b_{0} / 4\right)$ is the scattering phase shift associated with the potential $J_{K}^{z} S_{i}^{z} / 2, \rho_{0}=\left(2 \pi v_{F}\right)^{-1}$ is the conduction electron density of states at the Fermi energy, and $b_{0}$ is the lattice parameter in the fermionic chain. For simplicity we assume these parameters to be identical for all chains. We then introduce the (Emery-Kivelson) unitary transformation: ${ }^{.54}$

$$
\mathcal{U}=\exp \left[-i \gamma \sum_{i} S_{i}^{z} \phi_{i, s}^{R}(0)\right],
$$

under which the bosonic field $\nabla \phi_{i, s}^{R}(y)$ and the spin operator $S_{i}^{+}$transform as

$$
\begin{gathered}
\mathcal{U}^{\dagger} \nabla \phi_{i, s}^{R}(y) \mathcal{U}=\left[\nabla \phi_{i, s}^{R}(y)+\delta(y) 2 \pi \gamma S_{i}^{z}\right], \\
\mathcal{U}^{\dagger} S_{i}^{+} \mathcal{U}=S_{i}^{+} e^{i \gamma \phi_{i, s}^{R}(0)}
\end{gathered}
$$

Upon this transformation, the model Hamiltonian Eq. (1) transforms as $\tilde{\mathcal{H}}=\mathcal{U}^{\dagger} \mathcal{H} \mathcal{U}=\tilde{\mathcal{H}}_{F}+\tilde{\mathcal{H}}_{K}+\tilde{\mathcal{H}}_{X X Z}$, with

$$
\begin{gathered}
\tilde{\mathcal{H}}_{F}=\mathcal{H}_{F} \\
\tilde{\mathcal{H}}_{K}=\sum_{i}-\frac{2 \tilde{\delta}_{s}}{\pi \rho_{0}} S_{i}^{z} \frac{\nabla \phi_{i, s}^{R}(0)}{\sqrt{2} \pi} \\
+\sum_{i} \frac{J_{K}^{\perp} b_{0}}{2}\left[S_{i}^{+} \frac{e^{-i(\sqrt{2}-\gamma) \phi_{i, s}^{R}(0)}}{2 \pi b_{0}}+\text { H.c. }\right] \\
\tilde{\mathcal{H}}_{X X Z}=\sum_{i} J_{H}^{z} S_{i}^{z} S_{i+1}^{z}+\sum_{i} \frac{J_{H}^{\perp}}{2} e^{i \gamma\left[\phi_{i, s}^{R}(0)-\phi_{i+1, s}^{R}(0)\right]} S_{i}^{+} S_{i+1}^{-} \\
+ \text {H.c., }
\end{gathered}
$$

where we have defined $\tilde{\delta}_{s} \equiv \delta_{s}-\pi \gamma / 2 \sqrt{2}$. Note that in the transformed representation, the quantum dynamics of the bath [represented by the chiral field $\left.\phi_{i, s}^{R}(0)\right]$ appears explicitly in the Heisenberg term $\sim J_{H}^{\perp}\left(S_{i}^{+} S_{i+1}^{-} e^{i \gamma\left[\phi_{i, s}^{R}(0)-\phi_{i+1, s}^{R}(0)\right]}+\right.$ H.c. $)$. Physically, this means that the Heisenberg interaction is now "dressed" by the spin-density fluctuations of the electron gas. Note that the independent-bath model (19) is crucial to implement bosonization along the chains, and to put these ideas on a clear mathematical framework.

Up to now the parameter $\gamma$ appearing in Eq. (22) remains completely arbitrary. We now set $\gamma=\sqrt{2}$ in Eqs. (26) and (27), and the transformed Hamiltonians simplify to

$$
\begin{aligned}
\tilde{\mathcal{H}}_{K} & =\sum_{i}-\frac{2 \tilde{\delta}_{s}}{\pi \rho_{0}} S_{i}^{z} \frac{\nabla \phi_{i, s}^{R}(0)}{\sqrt{2} \pi}+\frac{J_{K}^{\perp}}{2 \pi} S_{i}^{x}, \\
\tilde{\mathcal{H}}_{X X Z}= & \sum_{i} J_{H}^{z} S_{i}^{z} S_{i+1}^{z} \\
& +\frac{J_{H}^{\perp}}{2} e^{i \sqrt{2}\left[\phi_{i, s}^{R}(0)-\phi_{i+1, s}^{R}(0)\right]} S_{i}^{+} S_{i+1}^{-}+\text {H.c. },
\end{aligned}
$$

where now $\tilde{\mathcal{H}}_{K}$ is equivalent to the spin-boson model with Ohmic dissipation, ${ }^{56-58}$ with $\tilde{\delta}_{s}$ related to the dissipative parameter $\alpha$ in the context of macroscopic quantum coherence through $\alpha=\left(2 \tilde{\delta}_{s} / \pi\right)^{2}$, and with the in-plane Kondo interaction playing the role of a magnetic field along the $x$ axis $h_{x}=$ $-J_{K}^{\perp} / 2 \pi$. That model describes a quantum phase transition from a phase with a "frozen" spin state (either $S_{i}^{z}=+1 / 2$ or $S_{i}^{z}=-1 / 2$ ) for $\alpha>1$, to a phase with an "untrapped" spin 
state for $\alpha<1$, where spin-flips induced by $S_{i}^{x}$ proliferate. The strong-coupling regime of the single-impurity Kondo Hamiltonian therefore corresponds to this last case, where (not too close to the transition) the Kondo temperature is $56,59,60$

$$
T_{K} \propto J_{K}^{\perp}\left(\frac{J_{K}^{\perp} b_{0}}{v_{F}}\right)^{\alpha /(1-\alpha)} .
$$

The special case $\alpha=0$ (i.e., $\tilde{\delta}_{s}=0$ ) was analyzed by Kotliar and $\mathrm{Si}$ in Ref. 55 and represents a particular limit where $\tilde{\mathcal{H}}_{K}$ can be diagonalized in the eigenbasis $| \pm\rangle_{i}$ of the operator $S_{i}^{x}$ (i.e., "Toulouse point II" in Ref. 55). Note that the condition $\tilde{\delta}_{s}=0$ implies that the original phase shift is $\delta_{s}=\pi / 2$, corresponding to the unitary limit $J_{K}^{z} b_{0} / v_{F} \rightarrow \infty$. Unfortunately, the unitary limit is not consistent with the well-known local Fermi-liquid description of the strong-coupling Kondo fixed point. ${ }^{43}$ Intuitively, in the limit $\tilde{\delta}_{S}=0$ the coupling to the bath vanishes and $\tilde{\mathcal{H}}_{K}$ reduces to a Zeeman Hamiltonian, which is not equivalent to the Kondo problem. ${ }^{15}$ However, as shown by Kotliar and $\mathrm{Si}$, a physically correct description of the strong-coupling limit is recovered by performing second-order perturbation expansion in $\tilde{\delta}_{s}{ }^{55}$ Physically, this is equivalent to reintroducing the coupling to the bath.

In the case of plane anisotropy, the Kondo couplings satisfy $J_{K}^{\perp}>J_{K}^{z}$. This implies that in order to perform an expansion around the point $\tilde{\delta}_{s}=0$, the microscopic parameters of the model should be in a regime such that strictly speaking the use of bosonization is not justified (i.e., the interactions are of the order or bigger than the Fermi energy). However, since this approach has been shown to successfully capture qualitatively the main features of the strong-coupling Kondo fixed point, ${ }^{55}$ we expect our approach to be correct only at a qualitative level. In addition, since $\tilde{\delta}_{s} \approx 0$ implies $\alpha \approx 0$, we will use Eq. (30) to identify $T_{K} \simeq J_{K}^{\perp}$ in what follows.

An effective low-energy Hamiltonian valid near $\tilde{\delta}_{s}=0$, and for the case where the Kondo interaction dominates over the Heisenberg exchange, that is, $J_{K}^{\perp} \gg\left\{J_{H}^{\perp}, J_{H}^{z}\right\}$ can be obtained expanding Eq. (28) at order $\tilde{\delta}_{s}^{2}$ and projecting onto the lowest energy level on each site $|-\rangle_{i}$,

$$
\tilde{\mathcal{H}}_{K}^{\prime} \equiv P_{-} \tilde{\mathcal{H}}_{K} P_{-}=-\sum_{i} \frac{1}{4}\left(\frac{2 \tilde{\delta}_{s}}{\pi \rho_{0}}\right)^{2}\left(\frac{2 \pi}{J_{K}^{\perp}}\right)\left(\frac{\nabla \phi_{i, s}^{R}(0)}{\sqrt{2} \pi}\right)^{2},
$$

where we have introduced the projector operator on the subspace spanned by $|-\rangle_{i}$ [i.e., $P_{-} \equiv \prod_{i}\left(|-\rangle_{i}\left\langle-\left.\right|_{i}\right)\right.$ ], and where we have neglected a constant term $J_{K}^{\perp} / 2 \pi$. The effective magnetic field $h_{x}$ opens a gap of size $\Delta=h_{x}$ in the spin excitation spectrum and consequently the spin degrees of freedom are "frozen" in the lowest energy configuration $|-\rangle_{i}$. In contrast, spin-density fluctuations in the bath remain gapless and their dynamics becomes dominant at low energies. Projecting $\tilde{\mathcal{H}}_{X X Z}$ onto this basis yields

$$
\begin{aligned}
\tilde{\mathcal{H}}_{X X Z}^{\prime} & \equiv P_{-} \tilde{\mathcal{H}}_{X X Z} P_{-} \\
& =\sum_{i} \frac{J_{H}^{\perp}}{4} \cos \sqrt{2}\left[\phi_{i, s}^{R}(0)-\phi_{i+1, s}^{R}(0)\right] .
\end{aligned}
$$

In this representation, the Heisenberg term $J_{H}^{\perp}\left(S_{i}^{+} S_{i+1}^{-}+\right.$H.c. $)$ induces an effective interaction between neighboring baths, encoded in the term $\sim \cos \sqrt{2}\left[\phi_{i, s}^{R}(0)-\phi_{i+1, s}^{R}(0)\right]$. This is an important result in our work, complementary to the situation analyzed in Sec. III A, where the opposite limit $J_{H}^{\perp} \gg J_{K}^{\perp}$ was studied. In that case, the bath was integrated out, and we studied the stability of the TLL phase to leading order in perturbation theory. Here, we do just the opposite: We keep the degrees of freedom of the bath and eliminate the spin degrees of freedom.

Although a general derivation of an effective low-energy model (i.e., for an arbitrary value of $\gamma$ ) is beyond the scope of the present work, it is worth noting that the effective coupling between fermionic baths that appears in Eq. (32) is a general physical feature that does not depend on our particular derivation for $\gamma=\sqrt{2}$. This can be understood using, for example, similar arguments as those leading to the Nozières' local Fermi liquid. ${ }^{43}$ Indeed, when $J_{K}^{\perp} \gg J_{H}^{\perp}$, a natural approach is to start from the Kondo singlets at neighboring sites $i$ and $i+1$, that is, $\left|G_{i}\right\rangle$ and $\left|G_{i+1}\right\rangle$, respectively, where $\left|G_{l}\right\rangle=\left(|\Uparrow\rangle_{l}\left|c_{l 0, \downarrow}^{\dagger}\right\rangle-|\Downarrow\rangle_{l}\left|c_{l 0, \uparrow}^{\dagger}\right\rangle\right) / \sqrt{2}$. The perturbation $\mathcal{H}^{\prime}=-t \sum_{l=\{i, i+1\}}\left(c_{l 1, \sigma}^{\dagger} c_{l 0, \sigma}+\right.$ H.c. $)$ acting on these neighboring singlets produces virtual excitations to the $n_{l 0}=1$ triplet subspace, at order $\left(t / J_{K}^{\perp}\right)^{2}$ on each one of them. Eventually, the Heisenberg interaction $J_{H}^{\perp}\left(S_{i}^{+} S_{i+1}^{-}+\right.$H.c. $)$ restores the initial singlet ground states $\left|G_{i}\right\rangle$ and $\left|G_{i+1}\right\rangle$ and, as a net result, virtual processes generate an effective spin interaction $\sim J_{H}^{\perp}\left(t / J_{K}^{\perp}\right)^{4}\left(c_{i, 1, \uparrow}^{\dagger} c_{i+1,1, \downarrow}+\right.$ H.c. $)$ between the second sites in the chains $i$ and $i+1$. Bosonizing this induced effective interaction yields a term of the form $\sim \cos \sqrt{2}\left[\phi_{i, s}^{R}(0)-\phi_{i+1, s}^{R}(0)\right]$, analogous to Eq. (32).

In order to derive an effective 1D model, we integrate out of the modes $\phi_{i, s}^{R}(y)$ for $y \neq 0$. This can be done exactly using the functional integral representation of the partition function, and generates a term of the form $\sim\left|\omega_{m}\right|$ in the effective action, which stems from the (Ohmic) dissipation induced by the coupling to local bath (cf. Refs. 15 and 61 for details). The resulting Euclidean action of the system reads

$$
\begin{gathered}
\mathcal{S}^{\prime}=\mathcal{S}_{0}^{\prime}+\mathcal{S}_{H}^{\prime} \\
\mathcal{S}_{0}^{\prime}=\sum_{i}\left[\sum_{\omega_{m}} \frac{\left|\omega_{m}\right|}{4 \pi \beta}\left|\varphi_{i}\left(i \omega_{m}\right)\right|^{2}+\int_{0}^{\beta} d \tau \frac{\left(\partial_{\tau} \varphi_{i}(\tau)\right)^{2}}{2 \pi E_{0}}\right] \\
\mathcal{S}_{H}^{\prime}=\sum_{i} \int_{0}^{\beta} d \tau \frac{J_{H}^{\perp}}{4} \cos \left[\varphi_{i}(\tau)-\varphi_{i+1}(\tau)\right]
\end{gathered}
$$

where we have defined the more compact notation for local field $\varphi_{i} \equiv \sqrt{2} \phi_{i, s}^{R}(y=0)$, and where we have used the equation of motion of chiral fields (i.e., $\partial_{\tau} \varphi_{i}-i v_{F} \nabla \varphi_{i}=0$ ) to express $\nabla \varphi_{i}$ in terms of $\partial_{\tau} \varphi_{i}$. In addition, in Eq. (34) we have defined the parameter:

$$
E_{0} \equiv \frac{J_{K}^{\perp}}{4 \tilde{\delta}_{s}^{2}}
$$

where the singularity at $\tilde{\delta}_{s}=0$ is a consequence of the unphysical unitary limit mentioned above.

Note that the effective model Eq. (33) is formally equivalent to the action of a 1D Josephson-junction array with local Ohmic dissipation, with $\varphi_{i}$ the phase of the superconducting order parameter at site $i, J_{H}^{\perp}$ the Josephson coupling, and $E_{0}$ the charging energy with respect to the ground. ${ }^{19,62}$ It can be also brought to a form equivalent to a $1 \mathrm{D} \mathrm{O}(2)$ dissipative 
quantum rotor model if we write it in terms of $\mathbf{N}_{i}(\tau)=$ $\left(\cos \varphi_{i}(\tau), \sin \varphi_{i}(\tau)\right){ }^{24,63-65}$ The fact that Hamiltonian Eq. (1) can be mapped (in the limit $J_{K}^{\perp}, J_{K}^{z} \gg J_{H}^{\perp}, J_{H}^{z}$ ) to these dissipative models is an important result of our work which shows interesting underlying connections between apparently different physical situations.

To appreciate the physical consequences of the effective model in Eq. (33), we concentrate on the transverse spin correlation function $\mathcal{C}^{+-}(n, \tau)=\left\langle T_{\tau} S_{i+n}^{+}(\tau) S_{i}^{-}(0)\right\rangle$. In the transformed representation, this correlation is evaluated as (cf. Appendix B) $\mathcal{C}^{+-}(n, \tau)=\left\langle T_{\tau} e^{i \varphi_{i+n}(\tau)} e^{-i \varphi_{i}(0)}\right\rangle$. Near the strong-coupling single-impurity Kondo limit $J_{K}^{\perp} \gg\left\{J_{H}^{\perp}, J_{H}^{z}\right\}$, we obtain the result [cf. Eq. (B14)] $\mathcal{C}^{+-}(0, \tau) \sim \tau^{-2}$, as expected for the local Fermi-liquid description of the Kondo problem. ${ }^{43,55}$ This slow decay is a consequence of the Ohmic dissipation term $\sim\left|\omega_{m}\right|$ in Eq. (34), inherited from the dynamics of the semi-infinite fermionic chain. This behavior is consistent with the strong-coupling Kondo picture where, at long times, the spin degrees of freedom are merged with those of the fermion bath. ${ }^{55}$ On the other hand, the exponentially decaying nonlocal correlation [cf. Eq. (B15)],

$$
\mathcal{C}_{n}^{+-}(n, 0)=\frac{1}{2} \frac{e^{-n / \xi_{c}}}{n+1},
$$

with $\xi_{c} \equiv 1 / \ln \left|\frac{8 e^{\gamma_{E}}}{\pi} \frac{E_{0}}{J_{H}^{\perp}}\right|$ the correlation length (where $\gamma_{E}=$ $0.577 \ldots$ is the Euler gamma constant), indicates that the spins are not spatially correlated beyond a distance $\xi_{c}$, supporting the idea that in this limit the spin chain realizes a disordered phase of nearly independent Kondo singlets.

\section{Hubbard-Stratonovich decoupling and RG analysis of the effective $\psi^{4}$ theory}

The properties and phases of action (33) can be investigated introducing an auxiliary bosonic field $\psi_{i}(\tau)$ to decouple the Heisenberg term $J_{H}^{\perp}$ (cf., e.g., Refs. 66-68):

$$
\begin{aligned}
& \frac{J_{H}^{\perp}}{4} \sum_{i} \int_{0}^{\beta} d \tau \cos \left[\varphi_{i}(\tau)-\varphi_{i+1}(\tau)\right] \\
& \rightarrow \int_{0}^{\beta} d \tau \sum_{i, j} \psi_{i}^{*}(\tau)\left[\mathbf{J}^{-\mathbf{1}}\right]_{i j} \psi_{j}(\tau) \\
& \quad-\frac{1}{2} \int_{0}^{\beta} d \tau \sum_{i}\left[\psi_{i}^{*}(\tau) e^{i \varphi_{i}(\tau)}+\psi_{i}(\tau) e^{-i \varphi_{i}(\tau)}\right],
\end{aligned}
$$

where we have defined the matrix $[\mathbf{J}]_{i j} \equiv \frac{1}{8} J_{H}^{\perp}\left(\delta_{i, j+1}+\delta_{i+1, j}\right)$. Then, the partition function reads $Z=Z_{0} \int \mathcal{D}[\psi] e^{-S[\psi]}$, where

$$
\begin{aligned}
\mathcal{S}[\psi]= & \sum_{i, j} \int_{0}^{1 / T} d \tau \psi_{i}^{*}(\tau)\left[\mathbf{J}^{-\mathbf{1}}\right]_{i j} \psi_{j}(\tau) \\
& -\ln \left\langle\exp \frac{1}{2} \sum_{i} \int_{0}^{1 / T} d \tau\left[\psi_{i}^{*}(\tau) e^{i \varphi_{i}(\tau)}+\text { H.c. }\right]\right\rangle_{0},
\end{aligned}
$$

is the effective action for the auxiliary field $\psi_{i}(\tau)$. Here, the notation $\langle\ldots\rangle_{0}$ means average with respect to the local action (34). A cumulant expansion of the last term in Eq. (39) to order

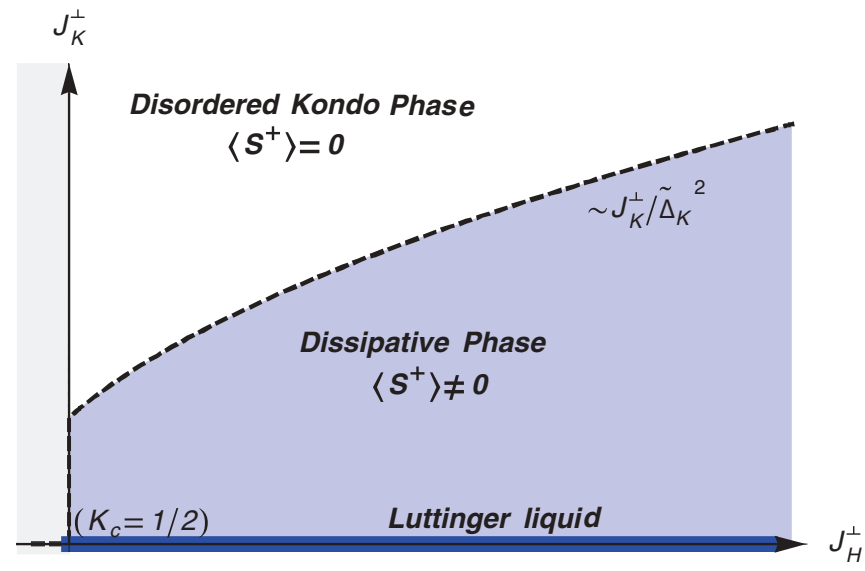

FIG. 3. (Color online) Schematic $T=0$ phase diagram of the dissipative 1D Kondo-Heisenberg spin chain. The thick blue line at $J_{K}^{\perp}=0$ corresponds to the Tomonaga-Luttinger liquid phase, which is unstable against a small perturbation $J_{K}^{\perp}$. The dashed line is the critical line separating a phase of disordered Kondo singlets (upper white area), from a dissipative phase (shaded bottom area), characterized by gapless diffusive spin excitations. In this phase, at $T=0$, the dissipative dynamics induced by the metallic environment stabilizes long-range order in the transverse magnetization.

$\psi^{4}$ yields

$\mathcal{S}[\psi]=\frac{T}{2 N_{i}} \sum_{\mathbf{q}} G_{0}^{-1}(\mathbf{q})\left|\psi_{\mathbf{q}}\right|^{2}+\frac{u}{4 !} \sum_{i} \int_{0}^{1 / T} d \tau\left|\psi_{i}(\tau)\right|^{4}$,

where the compact notation $\mathbf{q}=\left(k, \omega_{m}\right)$, with $\omega_{m} \equiv 2 \pi m T$ the bosonic Matsubara frequencies, ${ }^{69}$ has been used, and where $N_{i}$ is the number of spins. Here we have defined the Gaussian propagator,

$$
G_{0}^{-1}(\mathbf{q}) \equiv r+\frac{4}{J_{H}^{\perp}} k^{2}+\frac{\pi e^{-2 \gamma_{E}}}{E_{0}^{2}}\left|\omega_{m}\right|,
$$

where

$$
\begin{aligned}
r & \equiv \frac{8}{J_{H}^{\perp}}-\frac{1}{2} \int_{0}^{\beta} d \tau e^{i \omega_{m} \tau}\left\langle T_{\tau} e^{i \Phi_{m}\left(\tau_{1}\right)} e^{-i \Phi_{m}\left(\tau_{2}\right)}\right\rangle_{0} \\
& =\frac{8}{J_{H}^{\perp}}-\frac{\pi e^{-\gamma_{E}}}{2 E_{0}}, \\
u & \equiv \frac{c}{E_{0}^{3}},
\end{aligned}
$$

with $c=21.8 \ldots$ a numerical coefficient. At the mean-field level [i.e., the saddle-point approximation to Eq. (40)], this model describes a QPT when $J_{H}^{\perp}$ reaches the critical value $J_{H, c}^{\perp} \equiv 16 E_{0} / \pi e^{-\gamma_{E}} \propto J_{K}^{\perp} / \tilde{\delta}_{s}^{2}$. In Fig. 3 we show schematically this critical line as a dashed black line, separating the disordered phase with $\left\langle\psi_{i}\right\rangle=0$, corresponding to the disordered Kondo phase described in Sec. III B [cf. Eq. (37)], from the ordered phase with $\left\langle\psi_{i}\right\rangle \neq 0$. Physically, when $J_{H}^{\perp}>J_{H, c}^{\perp}$ the Heisenberg interaction is large enough to induce long-range coherence in the transverse magnetization $\left\langle S_{i}^{+}\right\rangle \propto$ $\left\langle e^{i \varphi_{i}}\right\rangle \propto\left\langle\psi_{i}\right\rangle$ along the spin chain. On the other hand, when $J_{H}^{\perp}<J_{H, c}^{\perp}$ the "charging" term $E_{0}$ induces large quantum 
fluctuations of the field $\varphi_{i}$ (i.e., local spin flips induced by the term $J_{K}^{\perp}$ ) and therefore tends to destroy the ordered state. In this representation, the competition between the couplings $J_{K}^{\perp}$ and $J_{H}^{\perp}$ becomes transparent.

Beyond the mean-field level, the quantum critical properties of this model, generalized to describe an $N$-component field $\left\{\psi_{\alpha}(x)\right\}=\left\{\psi_{1}(x), \psi_{2}(x), \ldots, \psi_{N}(x)\right\}$ in $d$ dimensions, are well known and have been studied in the context of antiferromagnetic instabilities of Fermi liquids, ${ }^{67,70}$ using the framework of the Hertz-Moriya-Millis theory. ${ }^{71-73}$ This theory describes quantum fluctuations of the order parameter $\psi_{\alpha}(x)$, with critical dynamical exponent $z=2$, around the Gaussian fixed point [i.e., $u=0$ in Eq. (40)]. A standard momentumshell RG procedure, performing a two-loop expansion in $u$, and in the small parameter $\epsilon=4-D$, where $D=d+z$, allows one to obtain the RG-flow equations for the parameters of model (40), ${ }^{67,70}$

$$
\begin{gathered}
\frac{d r(\ell)}{d \ell}=2 r(\ell)+u(\ell) \frac{N+2}{6} \frac{A_{D}}{1+r(\ell)}, \\
\frac{d u(\ell)}{d \ell}=\epsilon u(\ell)-u^{2}(\ell) \frac{N+8}{6} \frac{A_{D}}{(1+r(\ell))^{2}},
\end{gathered}
$$

where $A_{D}=2 \pi^{(D / 2)} / \Gamma(d / 2)$ [with $\Gamma(x)$ the Euler gamma function] is the surface area of the $D$-dimensional sphere, and where the units are such that the high-energy cutoff of the theory (40) is $\Lambda=1$. For $\epsilon$ small and positive $(d<2)$, this $\mathrm{RG}$ flow is controlled by a Wilson-Fisher fixed point located at $r^{*}=-\epsilon(N+2) / 2(N+8), u^{*}=6 \epsilon / A_{D}(N+8)$.

The stability of the Wilson-Fisher fixed point can be studied upon expansion of Eqs. (45) and (46) in the small deviations $\delta r=r-r^{*}, \delta u=u-u^{*}$, and allows one to obtain the eigencoupling equation $d w_{i} / d \ell=\lambda_{i} w_{i},(i=1,2)$, with eigenvalues $\lambda_{1}=2-\epsilon(N+2) /(N+8)+\mathcal{O}\left(\epsilon^{2}\right)$ and $\lambda_{2}=-\epsilon+\mathcal{O}\left(\epsilon^{2}\right)$, determining the critical exponents of the transition. ${ }^{67,70}$ Although for $d=1$ the parameter $\epsilon=4-$ $(d+z)=1$, which is not small, recent quantum Monte Carlo simulations ${ }^{65}$ have shown evidence of a QPT in $X Y$ spin chains subject to local Ohmic dissipation, with critical exponents in good agreement with those predicted using the $\epsilon$ expansion. ${ }^{65,70}$

In the ordered phase $\left\langle\psi_{i}\right\rangle \neq 0$ occurring for $J_{H}^{\perp}>J_{H, c}^{\perp}$, since the $U(1)$ symmetry of Eq. (39) is spontaneously broken, a Goldstone mode arises from smooth fluctuations of the phase of the order parameter $\psi_{i} \simeq\left|\psi_{i}\right| e^{i \vartheta_{i}}$, and it becomes necessary to check the stability of the ordered phase. To that end, we return to Eq. (40) and perform an expansion in small fluctuations of the phase $\delta \vartheta_{i}=\vartheta_{i}-\vartheta_{0}$, around an arbitrary value $\vartheta_{0}$. At Gaussian order in $\delta \vartheta_{i}$ we obtain the effective action,

$$
\begin{aligned}
& \mathcal{S}_{\text {eff }}[\vartheta]=\frac{1}{2} \frac{1}{\beta N_{i}} \sum_{\mathbf{q}} G_{\text {eff }}^{-1}(\mathbf{q})|\vartheta(\mathbf{q})|^{2}, \\
& G_{\text {eff }}^{-1}(\mathbf{q})=\frac{\pi e^{-2 \gamma_{E}} \psi_{0}^{2}}{E_{0}^{2}}\left|\omega_{m}\right|+\frac{4 \psi_{0}^{2}}{J_{H}^{\perp}} k^{2},
\end{aligned}
$$

where $G_{\text {eff }}^{-1}(\mathbf{q})$ is the propagator of the Goldstone mode, and where $\psi_{0}$ is the saddle-point solution of Eq. (40). This propagator describes a gapless phase characterized by diffusive $(z=2)$ excitations of the field $\vartheta(\mathbf{q})$, and by correlation functions $\mathcal{C}^{+-}(x, \tau) \equiv\left\langle T_{\tau} S^{+}(x, \tau) S^{-}(0)\right\rangle$ decaying as $\mathcal{C}^{+-}(x, 0) \sim$ $|x|^{-1}$ at long distances, and $\mathcal{C}^{+-}(\tau) \sim|\tau|^{-1 / 2}$ at long times (cf. Appendix B2). Using Eq. (47) to evaluate the average of the order parameter $\left\langle\psi_{i}\right\rangle=\psi_{0}\left\langle e^{i \vartheta_{i}}\right\rangle=\psi_{0} e^{-\frac{1}{2}\left\langle\vartheta_{i}^{2}\right\rangle}$, we obtain the result,

$$
\left\langle\psi_{i}\right\rangle=\psi_{0} \exp \left[-\left(\frac{e^{\gamma_{E}}}{2 \pi}\right)^{2} \frac{2 E_{0}}{\psi_{0}^{2}} \sqrt{J_{H}^{\perp} E_{0}}\right] \quad(\text { at } T=0) .
$$

Interestingly, due to the presence of the dissipative term $\sim\left|\omega_{m}\right|$, the (Gaussian) fluctuations of the spin chain are strongly suppressed relative to the isolated $(X Y)$ chain. Indeed, contrary to the case of isolated $1 \mathrm{D}$ systems, quantum fluctuations do not destroy the LRO because the effective dimensionality of the quantum system is $D=d+z=3$, larger than the critical dimension $D_{c}=2$ determined by the Gaussian theory for the Goldstone mode Eq. (47). Note that this is not in contradiction with the Mermin-Wagner theorem, ${ }^{74}$ which predicts the destruction of LRO at $T=0$ in 1D systems with short-range interactions. In our case, due to the presence of a higher-dimensional fermionic bath which induces long-ranged (imaginary) time correlations, the system cannot be considered strictly one-dimensional. Therefore, the Mermin-Wagner theorem is not applicable to our system.

At this point, it is interesting to note the connection with the weak-coupling TLL description of Sec. III A, which becomes apparent using, for instance, the self-consistent harmonic approximation (SCHA) method. ${ }^{75}$ This method consists of finding the optimal propagator $G_{\text {trial }}^{-1}(\mathbf{q})$ of a trial Gaussian action, such that the variational free energy of the system is minimized. For the case of Sec. III A, when $K>1 / 2$ the only relevant variable is the field $\Theta(x)$, describing the transverse spin excitations of the spin chain weakly coupled to the metal, and consequently the trial action writes compactly as $S_{\text {trial }}=$ $\frac{1}{2 \beta L} \sum_{\mathbf{q}} G_{\text {trial }}^{-1}(\mathbf{q})|\Theta(\mathbf{q})|^{2}$. Here we do not show the derivation of the SCHA equations, and refer the reader to Refs. 24 and 26, where this method was applied to closely related 1D TLL systems in contact to dissipative baths. The optimal propagator is $G_{\text {trial }}^{-1}(\mathbf{q})=\eta\left|\omega_{m}\right|+K\left(u k^{2}+\omega_{m}^{2} / u\right) / \pi$, with $\eta \sim J_{K}^{\perp}$. The similar forms (in the limit $\mathbf{q} \rightarrow 0$ ) of $G_{\text {trial }}^{-1}(\mathbf{q})$ and $G_{\text {eff }}^{-1}(\mathbf{q})$ in Eq. (48) suggests that the dissipative gapless phase with $z=2$, obtained in the strong-coupling regime, is also stable in the weak-coupling regime (see lower part of Fig. 3). The thick solid line at the bottom (i.e., $J_{K}^{\perp}=0$ ) corresponds to the gapless TLL phase described by the Hamiltonian (8). As shown by the RG flow Eq. (18), this line is unstable against a vanishingly small perturbation $J_{K}^{\perp}$.

\section{SUMMARY AND CONCLUSIONS}

In this work we have studied the quantum critical properties of a $S=1 / 2$ spin chain described by the anisotropic $X X Z$ Hamiltonian (5), coupled to a metallic environment via the anisotropic Kondo model (6). From the theoretical point of view, this model is related to the well-known Kondo-lattice model, which is relevant to the description of 
(higher-dimensional) heavy-fermion systems. ${ }^{9}$ In addition, it also encodes an interesting interplay between quantum spin fluctuations, enhanced by the one-dimensional geometry, and dissipation due to the Kondo coupling to the metallic environment. From the point of view of experimental systems, this is a relevant model for the description of linear arrangements of magnetic impurities built on the top of clean metallic surfaces by the means of STM techniques (see Fig. 1). ${ }^{3,14}$

We study the model Eq. (1) in different regimes of parameters using various analytical approaches (i.e., Abelian bosonization, renormalization-group method, analysis of the Ginzburg-Landau functional, etc.), and obtain the quantum phases at $T=0$.

There are two crucial assumptions in our work: (A) the presence of easy-plane anisotropy in $\mathcal{H}_{X X Z}$ and $\mathcal{H}_{K}$, that favors the couplings in the plane of the metallic surface. In real systems, this assumption is physically reasonable due to the presence of Dzyaloshinskii-Moriya interactions (induced by spin-orbit coupling at the surface of metals) which break the $S U(2)$ invariance, and enhance the transverse Heisenberg coupling $J_{H}^{\perp}$ [cf. Eq. (3)]. ${ }^{30,31}$ Other physical mechanisms producing other types of anisotropy, such as magnetocrystalline effects in the metallic host are beyond the scope of the present work. ${ }^{76,77}$ (B) The different dimensionality between the spin chain and the metallic environment, which allows one to assume the presence of enough Kondo-screening conduction electrons per spin in the chain.

In Sec. III A we set the stage by investigating the weakcoupling regime $J_{K}^{\perp} \ll J_{H}^{\perp}$ (see Sec. III A), where $\mathcal{H}_{K}$ can be considered a perturbation to the isolated spin chain. In that case, we derive a set of RG-flow equations [cf. Eqs. (16)-(18)] at first order in the parameters $J_{K}^{\perp}$ and $J_{K}^{z}$. This RG flow is determined by the value of the Luttinger parameter $K$ of the chain, and is drastically different from the RG flow expected for the single-impurity Kondo problem. ${ }^{9}$ Far away from the isotropic $S U(2)$ point $K=1 / 2$, while $J_{K}^{z}$ becomes an irrelevant coupling (in the RG sense), $J_{K}^{\perp}$ becomes relevant and destabilizes the Tomonaga-Luttinger liquid fixed point (cf. thick bottom line in Fig. 3). This situation is analogous to other TLL systems coupled to a dissipative environment. ${ }^{24,26}$ Closer to the symmetric point $K=1 / 2$, the effect of the irrelevant coupling $J_{K}^{z}$ becomes more important and higherorder perturbation analysis is required to study the RG flow of parameters. An important point about the scaling analysis of Sec. III A is that it indicates that the most relevant contribution (in the RG sense) to the free energy $\Delta F$ comes from the local sector of the spin response of the metal (this is discussed in detail in Appendix A). At this stage the only assumption made about the bath is that it has not been affected by (weak) coupling $J_{K}^{\perp}$.

In the opposite limit $J_{K}^{\perp} \gg J_{H}^{\perp}$ (cf. Sec. III B), we consider the problem starting from the limit of decoupled Kondoscreened impurities. Due to the higher dimensionality of the metallic host as compared to the spin chain, there are always sufficient conduction electrons to screen the impurity spins and the exhaustion problem ${ }^{51}$ never appears in the present situation. This allowed us to perform a crucial approximation, the "independent bath approximation," which amounts to neglect interference between neighboring Kondo clouds and to consider each spin as independently screened. Using this approximation, we show that the effective model for coupled Kondo impurities [cf. Eq. (33)] is formally equivalent to that of a 1D Josephson-junction array with on-site Ohmic dissipation, which is known to undergo a quantum phase transition as a function of the dissipation parameter. ${ }^{19}$ To extract the properties of the spin chain in this limit, we derive an effective Ginzburg-Landau theory [cf. Eq. (40)] in terms of a $U(1)$ bosonic Hubbard-Stratonovich field $\psi_{i}$, and study the critical properties around the Gaussian fixed point. ${ }^{67,70}$ Physically, the order parameter $\psi_{i}$ describes LRO in the transverse magnetization $\left\langle S_{i}^{+}\right\rangle \propto\left\langle\psi_{i}\right\rangle$ along the direction of the spin chain. In the limit $J_{K}^{\perp} \gg J_{H}^{\perp}$, the system is characterized by a vanishing order parameter $\left\langle\psi_{i}\right\rangle=0$, which we interpret as a manifestation of a disordered Kondo-singlet phase, where the spins are screened by their local fermionic bath. Upon the increase of $J_{H}^{\perp}$, the system experiences a QPT (dashed line in Fig. 3) towards a phase with LRO, characterized by a nonvanishing order parameter $\left\langle\psi_{i}\right\rangle \neq 0$ at $T=0$. Interestingly, the dynamics of the emerging Goldstone mode [cf. Eq. (47)] is not able to destroy the mean-field solution, which would be the usual situation for isolated 1D systems. This anomaly occurs due to the dissipative character of this mode.

It would be very interesting to verify our predictions on experimental level. Experimentally, the phase diagram could be studied with STM techniques either by varying the strength of the Kondo exchange coupling (i.e., by growing the 1D spin chain on the top of a decoupling layer ${ }^{3}$ ), or by changing the distance between spins, ${ }^{13}$ which has the effect of changing the magnitude and sign of the exchange interaction. More accurate predictions for the possible experimental observables are at present under progress. ${ }^{46}$

\section{ACKNOWLEDGMENTS}

The authors are grateful to T. Giamarchi, A. Georges, F. Guinea, M. Fabrizio, A. Iucci, and P. Simon for useful discussions. A.M.L. and P.C. acknowledge support from the Swiss National Science Foundation under MaNEP and Division II. A.M.L. acknowledges support from DARPA QuEST, JQI-NSFPFC. M.A.C. acknowledges the hospitality of D.-W. Wang at NCTS (Taiwan) and A. H. Castro-Neto at the Graphene Research Center of the National University of Singapore, and the support of Spanish MEC through Grant No. FIS2010-19609-C02-02.

\section{APPENDIX A: LEADING CORRECTIONS TO THE SPIN-CHAIN FREE ENERGY}

We begin by considering the partition function,

$$
Z=\operatorname{Tr} e^{-\left(\mathcal{H}_{0}+\mathcal{H}_{K}\right) / T}=Z_{0}\left\langle T_{\tau} e^{-\int_{0}^{1 / T} d \tau \mathcal{H}_{K}(\tau)}\right\rangle_{0},
$$

where $Z_{0} \equiv \operatorname{Tr} e^{-\mathcal{H}_{0} / T}$ is the partition function of the uncoupled host-chain system, and $\mathcal{H}_{0}=\mathcal{H}_{X X Z}+\mathcal{H}_{F}$ [cf. Eqs. (8) and (7)], and $\tau$ is the Matsubara imaginary time. ${ }^{69}$ The average 
$\langle\mathcal{A}\rangle_{0} \equiv \operatorname{Tr}\left[e^{-\mathcal{H}_{0} / T} \mathcal{A}\right] / \operatorname{Tr}\left[e^{-\mathcal{H}_{0} / T}\right]$ stands for the thermodynamic average of an operator $\mathcal{A}$ over the Gibbs ensemble defined by $\mathcal{H}_{0}$.

The free energy of the system (removing the bath contribution) is given by $F-F_{0}=-T \ln \left(Z / Z_{0}\right)=$
$-T \ln \left\langle T_{\tau} e^{-\int_{0}^{1 / T} d \tau \mathcal{H}_{K}(\tau)}\right\rangle_{0}$. Upon expanding the exponential to the lowest nontrivial order, we obtain the leading free-energy correction,

$$
\begin{aligned}
\Delta F & =-T \ln \left[1+\frac{1}{2 !} \int_{0}^{1 / T} d \tau_{1} d \tau_{2}\left\langle T_{\tau} \mathcal{H}_{K}\left(\tau_{1}\right) \mathcal{H}_{K}\left(\tau_{2}\right)\right\rangle_{0}+\cdots\right] \\
& =-\frac{T}{2} \int d \mathbf{r}_{1} d \mathbf{r}_{2}\left[\left(\frac{J_{K}^{z}}{k_{F}^{3}}\right)^{2}\left\langle T_{\tau} S^{z}\left(\mathbf{r}_{1}\right) S^{z}\left(\mathbf{r}_{2}\right)\right\rangle_{0} \chi^{z z}\left(\mathbf{r}_{12}\right)+2\left(\frac{J_{K}^{\perp}}{2 k_{F}^{3}}\right)^{2}\left\langle T_{\tau} S^{+}\left(\mathbf{r}_{1}\right) S^{-}\left(\mathbf{r}_{2}\right)\right\rangle_{0} \chi^{-+}\left(\mathbf{r}_{12}\right)\right]+\cdots,
\end{aligned}
$$

where we have introduced the compact notation $\mathbf{r} \equiv(x, \tau)$ and $\mathbf{r}_{12} \equiv \mathbf{r}_{1}-\mathbf{r}_{2}$. We have also defined the host spin-response function, $\chi^{a b}\left(\mathbf{R}_{1}-\mathbf{R}_{2}, \tau_{1}-\tau_{2}\right) \equiv\left\langle T_{\tau} s^{a}\left(\mathbf{R}_{1}, \tau_{1}\right) s^{b}\left(\mathbf{R}_{2}, \tau_{2}\right)\right\rangle_{0}-\chi_{0}^{a b}\left(\mathbf{R}_{1}-\mathbf{R}_{2}\right), \chi_{0}^{a b}\left(\mathbf{R}_{1}-\mathbf{R}_{2}\right)$ being the static part of the spin response of the host, which has been already included in the static spin-chain exchange couplings $J_{H}^{\perp}$ and $J_{H}^{z}$, determining the Luttinger parameter $K$. In deriving the above expression, we have used the $U(1) \times Z_{2}$ symmetry of the $X X Z$ chain, which implies that $\left\langle T_{\tau}\left[S^{+}\left(\mathbf{r}_{1}\right) S^{+}\left(\mathbf{r}_{2}\right)\right]\right\rangle_{0}=\left\langle T_{\tau}\left[S^{-}\left(\mathbf{r}_{1}\right) S^{-}\left(\mathbf{r}_{2}\right)\right]\right\rangle_{0}=0$, etc. Thus, even if the spin response of the metallic host lacks the in-plane spin-rotation $[U(1)]$ symmetry of the spin chain due to, for example, spin-orbit interactions [i.e., $\chi^{x y}(\mathbf{R}, \tau) \neq 0$, etc.], the leading corrections to the free energy are insensitive to it. However, one factor that complicates the weak-coupling analysis is the fact that the spin correlation functions of the host electrons have very different behavior as compared to the spin correlators of the uncoupled spin chain. In particular, the conformal invariance (e.g., homogeneous scaling upon space and time rescaling) present in the $X Y$ model [Eq. (8)] implies the spin correlators of the uncoupled chain read ${ }^{15}$

$$
\begin{aligned}
\left\langle T_{\tau} S^{z}(\mathbf{r}) S^{z}(0)\right\rangle_{0}= & -\frac{K}{4 \pi^{2}}\left[\frac{\left(\frac{\pi T}{u}\right)^{2}}{\sinh ^{2}\left(\frac{\pi T(x+i u \tau)}{u}\right)}+\text { H.c. }\right]+\frac{\cos \left(\frac{\pi x}{a_{0}}\right)}{a_{0}^{2} \pi} \frac{\left(\frac{\pi T a_{0}}{u}\right)^{2 K}}{\sinh ^{K}\left(\frac{\pi T(x+i u \tau)}{u}\right) \sinh ^{K}\left(\frac{\pi T(x-i u \tau)}{u}\right)}, \\
& \left\langle T_{\tau} S^{+}(\mathbf{r}) S^{-}(0)\right\rangle_{0}=\frac{\cos \left(\frac{\pi x}{a_{0}}\right)}{2 \pi a_{0}^{2}} \frac{\left(\frac{\pi T a_{0}}{u}\right)^{1 / 2 K}}{\sinh ^{\frac{1}{4 K}}\left(\frac{\pi T(x+i u \tau)}{u}\right) \sinh ^{\frac{1}{4 K}}\left(\frac{\pi T(x-i u \tau)}{u}\right)},
\end{aligned}
$$

where we have kept the leading terms at large distances and times. The uniform component of $S_{j}^{+}\left[\sim e^{-i \Theta(x)} \cos 2 \Phi(x)\right]$ is less relevant than the staggered part $\left[\sim e^{i x \pi / a_{0}} e^{-i \Theta\left(x_{j}\right)}\right]$ and will be neglected in what follows.

Estimating the temperature dependence of the various contributions to Eq. (A2) is possible analyzing the spinresponse function in the electron gas at long times and distances. In Fourier representation, this reduces to studying the polarization function $\chi^{a b}\left(\mathbf{Q}, \omega_{n}\right)$ near the $\operatorname{point}\left(\mathbf{Q}, \omega_{n}\right)=0$, with $\mathbf{Q}=\left(Q_{x}, Q_{y}, Q_{z}\right)$ the $3 \mathrm{D}$ wave vector in the metal. As is well known, ${ }^{69}$ this point is singular and consequently the limits $\mathbf{Q} \rightarrow 0, \omega_{n} \rightarrow 0$ do not commute, despite the fact that, in the present case, the singularity is expected to be smoothen by an integration over the momenta perpendicular to the spin chain. Consequently, we only need to retain the wave vector $Q_{x}$ parallel to the spin chain. Let us first study the response in the regime $Q_{x} \neq 0, \omega_{n} \rightarrow 0$. From general considerations, for a normal Fermi liquid, the long time dynamics in this regime is dominated by particle-hole excitations, which yield an Ohmic behavior:

$$
\chi_{x}^{a b}\left(Q_{x}, \omega_{n}\right) \propto f\left(Q_{x}\right)\left|\omega_{n}\right|,
$$

for a wide range of momentum transfer $Q_{x}<2 k_{F}$. Here we have defined $\chi_{x}^{a b}\left(Q_{x}, \omega_{n}\right) \equiv \int d Q_{y} d Q_{z} \chi^{a b}\left(\mathbf{Q}, \omega_{n}\right)$, and $f\left(Q_{x}\right)$ is a smooth function of $Q_{x}$ presenting no singularities. Thus, after Fourier transformation one obtains

$$
\chi_{x}^{a b}\left(Q_{x}, \tau \gg \omega_{c}^{-1}\right) \propto f\left(Q_{x}\right) \frac{T^{2}}{\sin ^{2}(\pi T \tau)},
$$

with $\omega_{c} \simeq E_{F}$. The lattice parameter of the spin chain $a_{0}$ determines a characteristic wave vector $Q_{x} \simeq a_{0}^{-1}$, and the result in Eq. (A6) can be interpreted as the Ohmic response arising from a slab of metal of width $\sim a_{0}$, constituting the "local" environment seen by each spin in the chain. This behavior has to be compared with the response in the opposite regime $\omega_{n} \neq 0, Q_{x} \rightarrow 0$,

$$
\chi_{x}^{a b}\left(Q_{x}, \omega_{n}\right) \propto g\left(\omega_{n}\right) Q_{x}^{2},
$$

which for an electron gas in the diffusive $\operatorname{limit}^{78}$ results in the stronger decay with distance,

$$
\chi_{x}^{a b}\left(x \gg a_{0}, \omega_{n}\right) \propto \frac{g\left(\omega_{n}\right)}{x^{3}} .
$$

Based on these qualitative arguments, we conclude that the most relevant contribution to $\Delta F$ arises from time decay in Eq. (A6), and we neglect the more irrelevant effects coming from Eq. (A8).

Using these approximations in Eq. (A2) yields

$$
\begin{aligned}
\frac{\Delta F}{L}= & g_{z, u}^{2} \frac{K A_{u}^{z}}{2^{6} \pi^{2} u^{2} k_{F}} T^{3} \\
& +g_{z, s}^{2} \frac{A_{s}^{z}}{2^{4} k_{F} a_{0}^{2} \pi^{3}}\left(\frac{\pi a_{0}}{u}\right)^{2 K} T^{1+2 K} \\
& +g_{\perp, s}^{2} \frac{A_{s}^{\perp}}{2^{5} k_{F} a_{0}^{2} \pi^{3}}\left(\frac{\pi a_{0}}{u}\right)^{1 / 2 K} T^{1+1 / 2 K},
\end{aligned}
$$


where we have defined the dimensionless couplings $g_{z, u} \equiv$ $J_{K}^{z} / v_{F} k_{F}, g_{z, s} \equiv J_{K}^{z} / v_{F} k_{F}$, and $g_{\perp, s} \equiv J_{K}^{\perp} / v_{F} k_{F}$, and where $A_{u}^{z}, A_{s}^{z}$, and $A_{s}^{\perp}$ are nonuniversal numerical coefficients.

\section{APPENDIX B: SPIN-SPIN CORRELATION FUNCTIONS}

Starting from the original spin representation (i.e., before the rotation $\mathcal{U})$, the transverse spin correlator is defined as

$$
\begin{aligned}
\mathcal{C}_{n}^{+-}(\tau) & \equiv\left\langle T_{\tau} S_{i+n}^{+}(\tau) S_{i}^{-}(0)\right\rangle_{\mathcal{H}} \\
& =\frac{\int \mathcal{D}[S] e^{-\mathcal{S}} e^{\mathcal{H} \tau} S_{i+n}^{+} e^{-\mathcal{H} \tau} S_{i}^{-}}{\int \mathcal{D}[S] e^{-\mathcal{S}}},
\end{aligned}
$$

where the average is taken with respect to the total Hamiltonian of the system Eq. (1). The correlation function is a physical quantity that does not depend on our particular choice of representation. Using the transformation $\mathcal{U}$, the Hamiltonian is transformed as in Eqs. (25) and (26), while the spin operators become $\mathcal{U}^{\dagger} S_{i}^{+} \mathcal{U}=S_{i}^{+} e^{i \gamma \phi_{i, \sigma}^{k}(0)}$ [see Eq. (24)]. Thus,

$$
\begin{aligned}
\mathcal{C}_{n}^{+-}(\tau)= & \left\langle T_{\tau}\left(\mathcal{U}^{\dagger} e^{\mathcal{H} \tau} \mathcal{U}\right)\left(\mathcal{U}^{\dagger} S_{i+n}^{+} \mathcal{U}\right)\right. \\
& \left.\times\left(\mathcal{U}^{\dagger} e^{-\mathcal{H} \tau} \mathcal{U}\right)\left(\mathcal{U}^{\dagger} S_{i}^{-} \mathcal{U}\right)\right\rangle
\end{aligned}
$$

$$
\begin{aligned}
& =\left\langle T_{\tau} e^{\tilde{\mathcal{H}} \tau} S_{i+n}^{+} e^{i \frac{\gamma}{\sqrt{2}} \varphi_{i+n}} e^{-\tilde{\mathcal{H}} \tau} S_{i}^{-} e^{-i \frac{\gamma}{\sqrt{2}} \varphi_{i}}\right\rangle \\
& =\left\langle T_{\tau} S_{i+n}^{+}(\tau) e^{i \frac{\gamma}{\sqrt{2}} \varphi_{i+n}(\tau)} S_{i}^{-}(0) e^{-i \frac{\gamma}{\sqrt{2}} \varphi_{i}(0)}\right\rangle_{\tilde{\mathcal{H}}}
\end{aligned}
$$

Imposing $\gamma=\sqrt{2}$, and near the strong-coupling Kondo fixed point, the Hamiltonian $\tilde{\mathcal{H}}$ maps onto Eqs. (31) and (32) (the conduction electron term $\mathcal{H}_{F}$ is not changed since it doesn't depend on spin operators). We therefore eliminate the spin degrees of freedom in the correlation function and obtain

$$
\begin{aligned}
\mathcal{C}_{n}^{+-}(\tau) & =\left\langle T_{\tau} e^{i \varphi_{i+n}(\tau)} e^{-i \varphi_{i}(0)}\right\rangle_{\tilde{\mathcal{H}}^{\prime}} \\
& =\frac{\int \mathcal{D}[\varphi] e^{-\mathcal{S}^{\prime}[\varphi]} e^{i \varphi_{i+n}(\tau)} e^{-i \varphi_{i}(0)}}{\int \mathcal{D}[\varphi] e^{-\mathcal{S}^{\prime}[\varphi]}},
\end{aligned}
$$

where the averages are now taken with respect to the effective action Eq. (33).

\section{Spin correlators in the disordered Kondo-singlet phase}

In the disordered "Kondo" phase $J_{K}^{\perp} \gg\left\{J_{H}^{\perp}, J_{H}^{z}\right\}$, the Heisenberg coupling $J_{H}^{\perp}$ in $\mathcal{S}_{H}^{\prime}$ Eq. (35) is a suitable expansion parameter to compute the correlation function. Explicitly

$$
\begin{aligned}
\mathcal{C}_{n}^{+-}(\tau) & =\frac{\int \mathcal{D}[\varphi] e^{-\mathcal{S}_{0}^{\prime}[\varphi]-\mathcal{S}_{H}^{\prime}[\varphi]} e^{i \varphi_{i+n}(\tau)} e^{-i \varphi_{i}(0)}}{\int \mathcal{D}[\varphi] e^{-\mathcal{S}_{0}^{\prime}[\varphi]-\mathcal{S}_{H}^{\prime}[\varphi]}} \\
& =\frac{\int \mathcal{D}[\varphi] e^{-\mathcal{S}_{0}}\left\{\sum_{m=0} \frac{1}{m !} \prod_{j=1}^{m}\left[\int_{0}^{\beta} d \tau_{j} \frac{J_{H}^{\perp}}{4^{2}}\left(\sum_{l} e^{i \varphi_{l+1}\left(\tau_{j}\right)-i \varphi_{l}\left(\tau_{j}\right)}+\text { H.c. }\right)\right]\right\} e^{i \varphi_{i+n}(\tau)-i \varphi_{i}(0)}}{\int \mathcal{D}[\varphi] e^{-\mathcal{S}_{0}[\varphi]}\left\{\sum_{m=0} \frac{1}{m !} \prod_{j=1}^{m}\left[\int_{0}^{\beta} d \tau_{j} \frac{J_{H}^{\perp}}{4^{2}}\left(\sum_{l} e^{i \varphi_{l+1}\left(\tau_{j}\right)-i \varphi_{l}\left(\tau_{j}\right)}+\text { H.c. }\right)\right]\right\}}, \\
& \simeq\left(\frac{J_{H}^{\perp}}{4^{2}}\right)^{n} \frac{1}{n !} \int_{0}^{\beta} d \tau_{1} d \tau_{2} \ldots d \tau_{n}\left\langle T_{\tau} e^{i \varphi_{i+n}(\tau)} e^{-i \varphi_{i+n}\left(\tau_{n}\right)} e^{i \varphi_{i+n-1}\left(\tau_{n}\right)} \ldots e^{-i \varphi_{i+1}\left(\tau_{1}\right)} e^{i \varphi_{i}\left(\tau_{1}\right)} e^{-i \varphi_{i}(0)}\right\rangle_{0},
\end{aligned}
$$

where we have truncated the perturbative expansion at leading order. This is to be expected, since the Heisenberg term only couples nearest neighbors, and therefore spins at a distance of $n$ sites only become correlated at order $\left(J_{H}^{\perp}\right)^{n}$ in the perturbative expansion. The product $\left\langle T_{\tau} e^{i \varphi_{i+n}(\tau)} e^{-i \varphi_{i+n}\left(\tau_{n}\right)} \ldots e^{-i \varphi_{i+1}\left(\tau_{1}\right)} e^{i \varphi_{i}\left(\tau_{1}\right)} e^{-i \varphi_{i}(0)}\right\rangle_{\mathcal{S}_{0}^{\prime}}$ can be calculated using Wick's theorem. For compactness in notation, we define the (local) two-point correlation function,

$$
\begin{aligned}
F(\tau) & \equiv\left\langle T_{\tau} e^{i \varphi_{m}\left(\tau_{1}\right)} e^{-i \varphi_{m}\left(\tau_{2}\right)}\right\rangle_{0} \\
& \simeq \frac{1}{\left(\frac{E_{0} \tau}{\sqrt{a}}\right)^{2}+1} \quad(\text { at } T=0),
\end{aligned}
$$

where $a$ is a numerical factor $a=4 e^{-2 \gamma_{E}} \simeq 1.261 \ldots$, and its Fourier transform,

$$
\begin{aligned}
F\left(\omega_{m}\right) & \equiv \int_{0}^{\beta} d \tau e^{i \omega_{m} \tau} F(\tau) \\
& =\frac{\pi e^{-\gamma_{E}}}{E_{0}} \exp \left[-\frac{\left|\omega_{m}\right| 2 e^{-\gamma_{E}}}{E_{0}}\right] .
\end{aligned}
$$

Then, the expression for $\mathcal{C}_{n}^{+-}(\tau)$ compactly writes

$$
\mathcal{C}_{n}^{+-}(\tau)= \begin{cases}\left(\frac{J_{H}^{\perp}}{2^{2}}\right)^{n} \frac{1}{\beta} \sum_{\omega_{m}} e^{i \omega_{m} \tau}\left[F\left(\omega_{m}\right)\right]^{n+1} . & n>0, \\ F(\tau) . & n=0,\end{cases}
$$

One particularly interesting case is the local dynamical correlation $\mathcal{C}_{0}^{+-}(\tau) \propto \tau^{-2}$ [cf. Eq. (B12)], which encodes the properties of a local Fermi liquid. Another one is the static, nonlocal correlation,

$$
\mathcal{C}_{n}^{+-}(0)=\frac{1}{2} \frac{e^{-n / \xi_{c}}}{n+1},
$$

where we have defined the correlation length $\xi_{c} \equiv$ $1 / \ln \left|\frac{8 e^{\gamma E}}{\pi} \frac{E_{0}}{J_{H}^{\perp}}\right|$.

\section{Spin correlations in the ordered phase}

In the ordered phase, the dynamics of the spin chain is effectively given by the action of the Goldstone mode Eq. (47). With this Gaussian action, and using the saddle-point equations in Eq. (39) to express $e^{i \varphi_{i}(\tau)}=\psi_{i}^{*}(\tau)$, we can calculate the spin correlation in Eq. (B6) as

$$
\begin{aligned}
\mathcal{C}_{n}^{+-}(\tau) & =\left\langle T_{\tau} e^{i \varphi_{i+n}(\tau)} e^{-i \varphi_{i}(0)}\right\rangle \\
& =(-1)^{n} \psi_{0}^{2}\left\langle T_{\tau} e^{-i \vartheta_{i+n}(\tau)} e^{i \vartheta_{i}(0)}\right\rangle \\
& =(-1)^{n} \psi_{0}^{2} e^{-\frac{1}{2}\left\langle T_{\tau}\left[\vartheta_{i+n}(\tau)-\vartheta_{i}(0)\right]^{2}\right\rangle},
\end{aligned}
$$

where the factor $(-1)^{n}$ comes from the antiferromagnetic correlations induced by the coupling $J_{H}^{\perp}>0,{ }^{79}$ and 
where

$$
\left\langle T_{\tau}\left[\vartheta_{i+n}(\tau)-\vartheta_{i}(0)\right]^{2}\right\rangle=\frac{1}{\beta L} \sum_{k, \omega_{m}} \frac{1-e^{i k a_{0} n-i \omega_{m} \tau}}{\frac{\pi}{e^{2 \gamma}} \frac{\psi_{0}^{2}\left|\omega_{m}\right|}{E_{0}^{2}}+\frac{16 \psi_{0}^{2} k^{2}}{J_{H}^{\perp}}} .
$$

We study this correlation function in two different limits: the local limit $n=0$ and $\tau=0$. In the first case we have

$$
\left\langle T_{\tau}\left[\vartheta_{i}(\tau)-\vartheta_{i}(0)\right]^{2}\right\rangle=\frac{1}{\beta L} \sum_{k, \omega_{m}} \frac{1-e^{-i \omega_{m} \tau}}{\frac{\pi}{e^{2 \gamma}} \frac{\psi_{0}^{2}\left|\omega_{m}\right|}{E_{0}^{2}}+\frac{16 \psi_{0}^{2} k^{2}}{J_{H}^{\perp}}},
$$

$$
\begin{aligned}
& =\left(\frac{1}{8 \pi}\right)^{2} \frac{J_{H}^{\perp}}{\psi_{0}^{2}} \int d \omega d k \frac{1-e^{-i \omega \tau}}{\frac{\pi}{16 e^{2 \gamma}} \frac{J_{H}^{\perp}|\omega|}{E_{0}^{2}}+k^{2}}, \\
& \simeq\left(\frac{1}{2 \pi}\right)^{2} \frac{\pi e^{\gamma}\left(J_{H}^{\perp}\right)^{3 / 2} E_{0}}{\sqrt{2} 8 \psi_{0}^{2}} \frac{1}{\sqrt{\tau}}, \quad \text { (B21) }
\end{aligned}
$$

where we have introduced the short-time cutoff $\tau_{0}$. With similar tools it can be shown that the static correlation decays as $\left\langle T_{\tau}\left[\vartheta_{i+n}(0)-\vartheta_{i}(0)\right]^{2}\right\rangle \sim|n|^{-1}$.
${ }^{1}$ R. Wiesendanger, Rev. Mod. Phys. 81, 1495 (2009).

${ }^{2}$ T. Jamneala, V. Madhavan, and M. F. Crommie, Phys. Rev. Lett. 87, 256804 (2001).

${ }^{3}$ C. F. Hirjibehedin, C. P. Lutz, and A. J. Heinrich, Science 312, 1021 (2006).

${ }^{4}$ L. Zhou, J. Wiebe, S. Lounis, E. Vedmedenko, F. Meier, S. Blügel, P. H. Dederichs, and R. Wiesendanger, Nature Physics 6, 187 (2010).

${ }^{5}$ D. Serrate, P. Ferriani, Y. Yoshida, S.-W. Hla, M. Menzel, K. von Bergmann, S. Heinze, A. Kubetzka, and R. Wiesendanger, Nature Nanotechnology 5, 350 (2010).

${ }^{6}$ J. Li, W.-D. Schneider, R. Berndt, and B. Delley, Phys. Rev. Lett. 80, 2893 (1998).

${ }^{7}$ V. Madhavan, W. Chen, T. Jamneala, M. F. Crommie, and N. S. Wingreen, Science 280, 567 (1998).

${ }^{8}$ N. Knorr, M. A. Schneider, L. Diekhoner, P. Wahl, and K. Kern, Phys. Rev. Lett. 88, 096804 (2002).

${ }^{9}$ A. C. Hewson, The Kondo Problem to Heavy Fermions (Cambridge University Press, Cambridge, 1993).

${ }^{10}$ M. A. Ruderman and C. Kittel, Phys. Rev. 96, 99 (1954).

${ }^{11}$ H. Löhneysen, J. Magn. Magn. Mater. 200, 532 (1999).

${ }^{12}$ M. N. Baibich, J. M. Broto, A. Fert, F. Nguyen Van Dau, F. Petroff, P. Etienne, G. Creuzet, A. Friederich, and J. Chazelas, Phys. Rev. Lett. 61, 2472 (1988).

${ }^{13}$ P. Wahl, P. Simon, L. Diekhöner, V. S. Stepanyuk, P. Bruno, M. A. Schneider, and K. Kern, Phys. Rev. Lett. 98, 056601 (2007).

${ }^{14}$ S. Loth, S. Baumann, C. P. Lutz, D. M. Eigler, and A. J. Heinrich, Science 335, 196 (2012).

${ }^{15}$ T. Giamarchi, Quantum Physics in One Dimension (Oxford University Press, Oxford, 2004).

${ }^{16}$ A. O. Caldeira and A. J. Leggett, Phys. Rev. Lett. 46, 211 (1981).

${ }^{17}$ G. Schön and A. D. Zaikin, Phys. Rep. 198, 237 (1990).

${ }^{18}$ R. Fazio and H. van der Zant, Phys. Rep. 355, 235 (2001).

${ }^{19}$ S. Tewari and J. Toner, Europhys. Lett. 74, 341 (2006).

${ }^{20}$ P. Goswami and S. Chakravarty, Phys. Rev. B 73, 094516 (2006).

${ }^{21}$ G. Refael, E. Demler, Y. Oreg, and D. S. Fisher, Phys. Rev. B 75, 014522 (2007).

${ }^{22}$ A. M. Lobos and T. Giamarchi, Phys. Rev. B 84, 024523 (2011).

${ }^{23}$ A. H. Castro Neto, C. de C. Chamon, and C. Nayak, Phys. Rev. Lett. 79, 4629 (1997).

${ }^{24}$ M. A. Cazalilla, F. Sols, and F. Guinea, Phys. Rev. Lett. 97, 076401 (2006).

${ }^{25}$ S. N. Artemenko and T. Nattermann, Phys. Rev. Lett. 99, 256401 (2007).

${ }^{26}$ A. M. Lobos, A. Iucci, M. Müller, and T. Giamarchi, Phys. Rev. B 80, 214515 (2009).
${ }^{27}$ A. M. Lobos and T. Giamarchi, Phys. Rev. B 82, 104517 (2010).

${ }^{28}$ P. P. Orth, I. Stanic, and K. Le Hur, Phys. Rev. A 77, 051601 (2008).

${ }^{29}$ V. S. Stepanyuk, A. N. Baranov, D. I. Bazhanov, W. Hergert, and A. A. Katsnelson, Surf. Sci. 482-485, 1045 (2001).

${ }^{30}$ C. Carbone, S. Gardonio, P. Moras, S. Lounis, M. Heide, G. Bihlmayer, N. Atodiresei, P. H. Dederichs, S. Blügel, S. Vlaic et al., Adv. Funct. Mater. 21, 1212 (2011).

${ }^{31}$ I. Garate and I. Affleck, Phys. Rev. B 81, 144419 (2010).

${ }^{32}$ E. Brezin and J. Zinn-Justin, editors, Fields, Strings and Critical Phenomena (Elsevier Science Publishers, Amsterdam, 1988).

${ }^{33}$ A. H. Castro Neto and B. A. Jones, Phys. Rev. B 62, 14975 (2000).

${ }^{34}$ Q. Si and F. Steglich, Science 329, 1161 (2010).

${ }^{35}$ O. Zachar and A. M. Tsvelik, Phys. Rev. B 64, 033103 (2001).

${ }^{36}$ O. Zachar, Phys. Rev. B 63, 205104 (2001).

${ }^{37}$ S. P. Strong and A. J. Millis, Phys. Rev. B 50, 9911 (1994).

${ }^{38}$ O. Zachar, S. A. Kivelson, and V. J. Emery, Phys. Rev. Lett. 77, 1342 (1996).

${ }^{39}$ A. E. Sikkema, I. Affleck, and S. R. White, Phys. Rev. Lett. 79, 929 (1997).

${ }^{40}$ H. Tsunetsugu, M. Sigrist, and K. Ueda, Rev. Mod. Phys. 69, 809 (1997).

${ }^{41}$ E. Novais, E. Miranda, A. H. Castro Neto, and G. G. Cabrera, Phys. Rev. Lett. 88, 217201 (2002).

${ }^{42}$ B. Braunecker, P. Simon, and D. Loss, Phys. Rev. Lett. 102, 116403 (2009).

${ }^{43}$ P. Nozières, J. Low Temp. Phys. 17, 31 (1974).

${ }^{44}$ M. Takahashi, Prog. Theor. Phys. 50, 1519 (1973).

${ }^{45}$ A. Luther and I. Peschel, Phys. Rev. B 12, 3908 (1975).

${ }^{46}$ A. M. Lobos, M. A. Cazalilla, and P. Chudzinski (unpublished).

${ }^{47}$ A. O. Gogolin, A. A. Nersesyan, and A. M. Tsvelik, Bosonization and Strongly Correlated Systems (Cambridge University Press, Cambridge, 1999).

${ }^{48}$ L. C. Andreani and H. Beck, Phys. Rev. B 48, 7322 (1993).

${ }^{49}$ V. Barzykin and I. Affleck, Phys. Rev. B 61, 6170 (2000).

${ }^{50}$ J. Simonin, arXiv:0708.3604v1 (2007).

${ }^{51}$ P. Nozières, Ann. Phys. (Paris) 10, 19 (1985)

${ }^{52}$ J. Simonin, Phys. Rev. B 73, 155102 (2006).

${ }^{53}$ P. Schlottmann, J. Phys. (Paris) C6, 1486 (1978).

${ }^{54}$ V. J. Emery and S. A. Kivelson, in Fundamental Problems in Statistical Mechanics VII: Proceedings of the 1993 Altenberg Summer School, edited by H. van Beijeren and M. E. Ernst (North Holland, Amsterdam, 1994).

${ }^{55}$ G. Kotliar and Q. Si, Phys. Rev. B 53, 12373 (1996).

${ }^{56}$ F. Guinea, V. Hakim, and A. Muramatsu, Phys. Rev. B 32, 4410 (1985). 
${ }^{57}$ A. J. Leggett, S. Chakravarty, A. T. Dorsey, M. P. A. Fisher, A. Garg, and W. Zwerger, Rev. Mod. Phys. 59, 1 (1987).

${ }^{58}$ U. Weiss, Quantum Dissipative Systems (2nd edition), Vol. 10 (World Scientific Publishing, Singapore, 1999).

${ }^{59}$ F. Guinea, E. Bascones, and M. J. Calderon, AIP Conf. Proc. 438, 1 (1997)

${ }^{60}$ K. Le Hur, Ann. Phys. 323, 2208 (2008).

${ }^{61}$ A. O. Gogolin, A. A. Nersesyan, and A. M. Tsvelik, Bosonization and Strongly Correlated Systems (Cambridge University Press, Cambridge, 1999).

${ }^{62}$ This is in contrast to spins interacting with a $1 \mathrm{D}$ metal, where the single-impurity limit is only reached at much larger distances $d \gg \xi_{K}$, with $\xi_{K}=\hbar v_{F} / k_{B} T_{K}$ the Kondo correlation length and $T_{K}$ the Kondo temperature.

${ }^{63}$ S. Drewes, D. P. Arovas, and S. Renn, Phys. Rev. B 68, 165345 (2003).

${ }^{64}$ S. R. Renn, arXiv:cond-mat/9708194v1 (1995).

${ }^{65}$ P. Werner, M. Troyer, and S. Sachdev, J. Phys. Soc. Jpn. 74, 67 (2005).

${ }^{66}$ M. V. Feigel'man and A. I. Larkin, Chem. Phys. 235, 107 (1998).
${ }^{67}$ S. Sachdev, Quantum Phase Transitions (Cambridge University Press, Cambridge, 2000).

${ }^{68}$ R. M. Lutchyn, V. Galitski, G. Refael, and S. Das Sarma, Phys. Rev. Lett. 101, 106402 (2008).

${ }^{69}$ G. D. Mahan, Many-Particle Physics, Physics of Solids and Liquids, 3rd ed. (Kluwer Academic/Plenum Publishers, New York, 2000).

${ }^{70}$ S. Pankov, S. Florens, A. Georges, G. Kotliar, and S. Sachdev, Phys. Rev. B 69, 054426 (2004).

${ }^{71}$ J. A. Hertz, Phys. Rev. B 14, 1165 (1976).

${ }^{72}$ T. Moriya and J. Kawabata, J. Phys. Soc. Jpn. 34, 639 (1973).

${ }^{73}$ A. J. Millis, Phys. Rev. B 48, 7183 (1993).

${ }^{74}$ N. D. Mermin and H. Wagner, Phys. Rev. Lett. 17, 1133 (1967).

${ }^{75}$ R. P. Feynman, Statistical Mechanics (Benjamin, Reading, 1972).

${ }^{76}$ C. F. Hirjibehedin, C.-Y. Lin, A. F. Otte, M. Ternes, C. P. Lutz, B. A. Jones, and A. J. Heinrich, Science 317, 1199 (2007).

${ }^{77}$ M. A. Barral, P. Roura-Bas, A. M. Llois, and A. A. Aligia, Phys. Rev. B 82, 125438 (2010).

${ }^{78}$ E. Akkermans and G. Montambaux, Mesoscopic Physics of Electrons and Photons (Cambridge University Press, Cambridge, 2007).

${ }^{79}$ Strictly speaking, for the analogy to be correct, $J_{H}^{\perp}$ should have the opposite sign. This can be done introducing the trivial change of variables $\varphi_{i}=\varphi_{i}^{\prime}+i \pi$. 